Satizabal et al.

\title{
Genetic Architecture of Subcortical Brain Structures in 0ver 40,000 Individuals Worldwide
}

Claudia L Satizabal ${ }^{* 1,2}$, Hieab HH Adams*3,4, Derrek P Hibar*5, Charles C White*6,7, Jason L Stein ${ }^{5,8}$, Markus Scholz ${ }^{9,10}$, Murali Sargurupremraj ${ }^{11}$, Neda Jahanshad ${ }^{5}$, Albert V Smith ${ }^{12,13,14}$, Joshua C Bis ${ }^{15}$,

Xueqiu Jian'16, Michelle Luciano17, Edith Hofer18,19, Alexander Teumer20, Sven J van der Lee3, Jingyun Yang21,22, Lisa R Yanek23, Tom V Lee ${ }^{24}$, Shuo Li25, Yanhui Hu26, Jia Yu Koh27, John D Eicher28, Sylvane Desrivières $^{29}$, Alejandro Arias-Vasquez ${ }^{30,31,32,33}$, Ganesh Chauhan ${ }^{11,34}$, Lavinia Athanasiu ${ }^{35,36}$, Miguel E Renteria37, Sungeun Kim ${ }^{38,39,40}$, David Höhn"11, Nicola J Armstrong ${ }^{42}$, Qiang Chen ${ }^{43}$, Avram J Holmes ${ }^{44,45}$, Anouk den Braber ${ }^{46}$, Iwona Kloszewska ${ }^{47}$, Micael Andersson ${ }^{48,49}$, Thomas Espeseth ${ }^{35,50,}$ Oliver Grimm ${ }^{51}$, Lucija Abramovic 52 , Saud Alhusaini ${ }^{53,54}$, Yuri Milaneschi55, Martina Papmeyer56,57, Tomas Axelsson ${ }^{58}$, Stefan Ehrlich ${ }^{45,59,60}$, Roberto Roiz-Santiañez ${ }^{61,62}$, Bernd Kraemer ${ }^{63}$, Asta K Håberg64,65, Hannah J Jones 66,67,68, G Bruce Pike69, Dan J Stein70,71, Allison Stevens ${ }^{60}$, Janita Bralten $^{31,33}$, Meike W Vernooij ${ }^{3,4}$, Tamara B Harris ${ }^{72}$, Irina Filippi ${ }^{29}$, A Veronica Witte ${ }^{73,74}$, Tulio Guadalupe ${ }^{75,76}$, Katharina Wittfeld 77,78 , Thomas H Mosley9, James T Becker ${ }^{80}$, Nhat Trung Doan ${ }^{36}$, Saskia P Hagenaars ${ }^{17}$, Yasaman Saba ${ }^{81}$, Gabriel Cuellar-Partida ${ }^{82}$, Najaf Amin³ ${ }^{3}$ Saima Hilal83,84, Kwangsik Nho ${ }^{38,39,40}$, Nazanin Karbalai41, Konstantinos Arfanakis ${ }^{21,85,86}$, Diane M Becker23, David Ames $^{87,88}$, Aaron L Goldman ${ }^{43}$, Phil H Lee $45,89,90,91,92$, Dorret I Boomsma ${ }^{46}$, Simon Lovestone ${ }^{93,94}$, Sudheer Giddaluru95,96, Stephanie Le Hellard95,96, Manuel Mattheisen97,98,99,100,101, Marc M Bohlken ${ }^{52}$, Dalia Kasperaviciute102, Lianne Schmaal55,103,104, Stephen M Lawrie56, Ingrid Agartz ${ }^{36,100,105, ~ E s t h e r ~}$ Walton59,67, Diana Tordesillas-Gutierrez ${ }^{62,106}$, Gareth E Davies ${ }^{107}$, Jean Shin ${ }^{108}$, Jonathan C Ipser70, Louis N Vinke109, Martine Hoogman ${ }^{31,33}$, Maria J Knol³, Tianye Jia29, Ralph Burkhardt10,110, Marieke Klein ${ }^{31,33}$, Fabrice Crivello111, Deborah Janowitz 77 , Owen Carmichael112, Unn K Haukvik113,114, Benjamin S Aribisala115,116, Helena Schmidt81, Lachlan T Strike82,117, Ching-Yu Cheng27,118, Shannon L 
Risacher39,40, Benno Pütz ${ }^{41}$, Debra A Fleischman²1,22,119, Amelia A Assareh120, Venkata S

Mattay43,121,122, Randy L Buckner45,123, Patrizia Mecocci124, Anders M Dale125,126, Sven Cichon'127,128,129, Marco P Boks 52 , Mar Matarin102,130, Brenda WJH Penninx ${ }^{55}$, Vince D Calhoun 131,132, M Mallar Chakravarty133,134, Andre Marquand ${ }^{33,135}$, Christine Macare ${ }^{29}$, Shahrzad Kharabian Masouleh73,136, Jaap Oosterlaan137,138,139, Philippe Amouyel140, Katrin Hegenscheid141, Jerome I Rotter142, Andrew J Schork ${ }^{143,144}$, David CM Liewald17, Greig I De Zubicaray145, Tien Yin Wong27,118, Li Shen 38,39,40, Philipp G Sämann¹, Henry Brodaty120,146, Joshua L Roffman45, Eco JC De Geus6, Magda Tsolaki147, Susanne Erk $^{148}$, Kristel R Van Eijk ${ }^{149}$, Gianpiero L Cavalleri150, Nic JA Van der Wee ${ }^{151}$, Andrew M McIntosh ${ }^{17,56,}$ Randy L Gollub45,60,89, Kazima B Bulayeva152, Manon Bernard108, Jennifer S Richards ${ }^{30,33,153}$, Jayandra J Himali1,2,25, Markus Loeffler9,10, Nanda Rommelse ${ }^{32,33,154}$, Wolfgang Hoffmann"78,155, Lars T Westlye ${ }^{35,156,}$ Maria C Valdés Hernández ${ }^{115,157}$, Narelle K Hansell82,117, Theo GM Van Erp ${ }^{158,}$ Christiane Wolf159, John BJ Kwok ${ }^{160,161,162}$, Bruno Vellas ${ }^{163}$, Andreas Heinz ${ }^{164}$, Loes M Olde Loohuis ${ }^{165}$, Norman Delanty54,166, Beng-Choon Ho167, Christopher RK Ching5,168, Elena Shumskaya31,33,135, 169, Albert Hofman ${ }^{3,170}$, Dennis Van der Meer171, Georg Homuth ${ }^{172}$, Bruce M Psaty ${ }^{173,174}$, Mark Bastin 115,157, Grant W Montgomery82, Tatiana M Foroud ${ }^{40,175}$, Simone Reppermund ${ }^{120,176}$, Jouke-Jan Hottenga46, Andrew Simmons ${ }^{177,178,179}$, Andreas Meyer-Lindenberg51, Wiepke Cahn" ${ }^{52}$, Christopher D Whelan ${ }^{5,54}$, Marjolein MJ Van Donkelaar31,33, Qiong Yang25, Norbert Hosten ${ }^{141}$, Robert C Green ${ }^{89,180,}$ Anbupalam Thalamuthu120, Sebastian Mohnke148, Hilleke E Hulshoff Pol52, Honghuang Lin²,181, Clifford R Jack Jr182, Peter R Schofield161,183, Thomas W Mühleisen"129,184, Pauline Maillard169, Steven G Potkin158, Wei Wen 120, Evan Fletcher169, Arthur W Toga185, Oliver Gruber63, Matthew Huentelman ${ }^{186}$, George Davey Smith67, Lenore J Launer72, Lars Nyberg48,49,187, Erik G Jönsson³6,100, Benedicto CrespoFacorro ${ }^{61,62}$, Nastassja Koen 70,71 , Douglas Greve ${ }^{60,188}$, André G Uitterlinden³, Daniel R Weinberger43,189, Vidar M Steen ${ }^{95,96}$, Iryna O Fedko ${ }^{190}$, Nynke A Groenewold70, Wiro J Niessen 4,191,192, Roberto Toro ${ }^{193}$, Christophe Tzourio ${ }^{11}$, William T Longstreth Jr194, M Kamran Ikram,118, Jordan W 
Smoller45,89,91,92, Marie-Jose Van Tol195, Jessika E Sussmannn ${ }^{56}$, Tomas Paus ${ }^{196}$, Hervé Lemaître ${ }^{197}$, Bernard Mazoyer ${ }^{111}$, Ole A Andreassen ${ }^{35,36}$, Florian Holsboer ${ }^{41,198}$, 199, Dick J Veltman ${ }^{55}$, Jessica A Turner132,200, Zdenka Pausova ${ }^{108}$, Gunter Schumann²9, Daan Van Rooij30,33,201, Srdjan Djurovic ${ }^{95,202,}$ Ian J Deary ${ }^{17}$, Katie L McMahon²03, Bertram Müller-Myhsok¹,204,205, Rachel M Brouwer ${ }^{52}$, Hilkka Soininen206,207, Massimo Pandolfo199, Thomas H Wassink167, Joshua W Cheung5, Thomas Wolfers ${ }^{31,33}$, Jean-Luc Martinot'197, Marcel P Zwiers ${ }^{33,135}$, Matthias Nauck 208,209 , Ingrid Melle 35,36, Nicholas G Martin ${ }^{82}$, Ryota Kanai210,211,212, Eric Westman²13, René S Kahn², Sanjay M Sisodiya ${ }^{102}$, Tonya White ${ }^{4,214}$, Arvin Saremi5 ${ }^{5}$, Hans van Bokhoven ${ }^{31,33}$, Han G Brunner31,33,215,216, Henry Völzke20,209, Margaret J Wright117,203, Dennis Van 't Ent46, Markus M Nöthen128,217, Roel A Ophoff52,165, Jan K Buitelaar30,33,154, Guillén Fernández ${ }^{30,33}$, Perminder S Sachdev²0,218, Marcella Rietschel51, Neeltje EM Van Haren ${ }^{52}$, Simon E Fisher 33,76 , Alexa S Beiser ${ }^{2,25}$, Clyde Francks ${ }^{33,76}$, Andrew J Saykin ${ }^{39,40,175}$, Karen A Mather120, Nina Romanczuk-Seiferth ${ }^{164}$, Catharina A Hartman ${ }^{201}$, Anita L DeStefano ${ }^{2,25}$, Dirk J Heslenfeld $^{219}$, Michael W Weiner220,221, Henrik Walter ${ }^{148}$, Pieter J Hoekstra ${ }^{201}$, Paul A Nyquist ${ }^{23}$, Barbara Franke 31,32,33, David A Bennett21,22, Hans J Grabe 77,78, Andrew D Johnson28, Christopher Chen ${ }^{83,84}$, Cornelia M van Duijn ${ }^{3,222}$, Oscar L Lopez ${ }^{223}$, Myriam Fornage ${ }^{224}$, Joanna A Wardlaw17,157,225, Reinhold Schmidt18, Charles DeCarli226, Philip L De Jager7,227, Arno Villringer73,74, Stéphanie Debette11, Vilmundur Gudnason13,14, Sarah E Medland82**, Joshua M Shulman228,229**, Paul M Thompson ${ }^{5 * *}$, Sudha Seshadri ${ }^{1,2 * *}$, M Arfan Ikram ${ }^{3,4 * *}$

1. Department of Neurology, Boston University School of Medicine, Boston, Massachusetts, 02118, USA.

2. The Framingham Heart Study, 73 Mt Wayte Ave, Framingham, Massachusetts, 01702, USA.

3. Department of Epidemiology, Erasmus MC, Rotterdam, 3015 CE, The Netherlands. 
4. Department of Radiology and Nuclear Medicine, Erasmus MC, Rotterdam, 3015 CE, The Netherlands.

5. Imaging Genetics Center, USC Mark and Mary Stevens Neuroimaging \& Informatics Institute, Keck School of Medicine of University of Southern California, Los Angeles, 90292, USA.

6. Program in Translational NeuroPsychiatric Genomics, Institute for the Neurosciences, Departments of Neurology and Psychiatry, Brigham and Women's Hospital, Boston, Massachusetts, USA.

7. Program in Medical and Population Genetics, Broad Institute, Cambridge, Massachusetts, USA.

8. Department of Genetics \& UNC Neuroscience Center, University of North Carolina (UNC), Chapel Hill, North Carolina, 27599, USA.

9. Institute for Medical Informatics, Statistics and Epidemiology, University of Leipzig, 04107 Leipzig, Germany.

10. LIFE - Leipzig Research Center for Civilization Diseases, University of Leipzig, Leipzig, Germany.

11. Inserm U1219, University of Bordeaux, Bordeaux University Hospital, Bordeaux, France.

12. Department of Biostatistics, University of Michigan, Ann Arbor, Michigan, 48109, USA.

13. Faculty of Medicine, University of Iceland, 101.

14. Icelandic Heart Association, 201 Kopavogur, Iceland.

15. Cardiovascular Health Research Unit, Department of Medicine, University of Washington, 1730 Minor Avenue / Suite 1360 / Seattle, Washington 98101, USA.

16. The University of Texas Health Science Center at Houston, Houston, Texas, 77030, USA.

17. Centre for Cognitive Ageing and Cognitive Epidemiology, Psychology, University of Edinburgh, Edinburgh, EH8 9JZ, UK. 
18. Clinical Division of Neurogeriatrics, Department of Neurology, Medical University of Graz, Austria.

19. Institute for Medical Informatics, Statistics and Documentation, Medical University of Graz, Austria.

20. Institute for Community Medicine, University Medicine Greifswald, Greifswald, 17489, Germany.

21. Rush Alzheimer's Disease Center, Rush University Medical Center, Chicago, Illinois, 60612, USA.

22. Department of Neurological Sciences, Rush University Medical Center, Chicago, Illinois, 60612, USA.

23. GeneSTAR Research Program, Department of Medicine, Johns Hopkins University School of Medicine, Baltimore, MD 21287, USA.

24. Department of Neurology, Baylor College of Medicine, Houston, Texas 77030, USA.

25. Department of Biostatistics, Boston University School of Public Health, Boston, Massachusetts, 02118, USA.

26. Department of Genetics, Harvard Medical School, Boston, Massachusetts, USA.

27. Singapore Eye Research Institute, Singapore National Eye Centre, Singapore, 168751, Singapore.

28. National Heart, Lung and Blood Institute's The Framingham Heart Study, Division of Intramural Research, Population Sciences Branch, Framingham, Massachusetts, 01702, USA.

29. MRC-SGDP Centre, Institute of Psychiatry, Psychology and Neuroscience, King's College London, London, SE5 8AF, UK.

30. Department of Cognitive Neuroscience, Radboud University Medical Center, Nijmegen, The Netherlands. 
31. Department of Human Genetics, Radboud University Medical Center, Nijmegen, The Netherlands.

32. Department of Psychiatry, Radboud University Medical Center, Nijmegen, The Netherlands.

33. Donders Institute for Brain, Cognition and Behaviour, Radboud University, Nijmegen, The Netherlands.

34. Centre for Brain Research, Indian Institute of Science, Bangalore, India.

35. NORMENT - KG Jebsen Centre, Division of Mental Health and Addiction, Oslo University Hospital, Oslo, 0315, Norway.

36. NORMENT - KG Jebsen Centre, Institute of Clinical Medicine, University of Oslo, Oslo, 0315, Norway.

37. Department of Genetics and Computational Biology, QIMR Berghofer Medical Research Institute, Brisbane, QLD, Australia.

38. Center for Computational Biology and Bioinformatics, Indiana University School of Medicine, Indianapolis, Indiana, 46202, USA.

39. Center for Neuroimaging, Radiology and Imaging Sciences, Indiana University School of Medicine, Indianapolis, Indiana, 46202, USA.

40. Indiana Alzheimer Disease Center, Indiana University School of Medicine, Indianapolis, Indiana, 46202, USA.

41. Max Planck Institute of Psychiatry, Munich, 80804, Germany.

42. Mathematics and Statistics, Murdoch University, Perth, Western Australia, 6150, Australia.

43. Lieber Institute for Brain Development, Baltimore, 21205, USA.

44. Department of Psychology, Yale University, New Haven, 06520, USA.

45. Department of Psychiatry, Massachusetts General Hospital, Boston, Massachusetts 02114, USA. 
46. Biological Psychology, Neuroscience Campus Amsterdam, Vrije Universiteit \& Vrije Universiteit Medical Center, Amsterdam, 1081 BT, The Netherlands.

47. Medical University of Lodz, Lodz, 90-419, Poland.

48. Department of Integrative Medical Biology and Umeå center for Functional Brain Imaging, Umeå University, Umeå 90187 , Sweden.

49. Umeå Centre for Functional Brain Imaging (UFBI), Umeå University, Umeå 901 87, Sweden.

50. Department of Psychology, University of Oslo, Oslo, 0373, Norway.

51. Central Institute of Mental Health, Medical Faculty Mannheim, University Heidelberg, Mannheim, 68159, Germany.

52. Brain Center Rudolf Magnus, Department of Psychiatry, UMC Utrecht, Utrecht, 3584 CX, The Netherlands.

53. Department of Neurology and Neurosurgery, Montreal Neurological Institute, McGill University, Montreal, H3A 2B4, Canada.

54. The Royal College of Surgeons in Ireland, 123 St Stephen's Green, Dublin 2, Ireland.

55. Department of Psychiatry, Amsterdam Neuroscience, VU University Medical Center, Amsterdam, 1007 MB, The Netherlands

56. Division of Psychiatry, Royal Edinburgh Hospital, University of Edinburgh, Edinburgh, EH10 $5 \mathrm{HF}, \mathrm{UK}$.

57. Division of Systems Neuroscience of Psychopathology, Translational Research Center, University Hospital of Psychiatry, University of Bern, Switzerland.

58. Department of Medical Sciences, Molecular Medicine and Science for Life Laboratory, Uppsala University, Box 1432, SE-751 44 Uppsala, Sweden.

59. Division of Psychological and Social Medicine and Developmental Neurosciences, Faculty of Medicine, TU Dresden, 01307 Germany. 
60. Martinos Center for Biomedical Imaging, Massachusetts General Hospital, Charlestown, Massachusetts 02129, USA.

61. Department of Medicine and Psychiatry, University Hospital Marqués de Valdecilla, School of Medicine, University of Cantabria-IDIVAL, 39008 Santander, Spain.

62. CIBERSAM (Centro Investigación Biomédica en Red Salud Mental), Santander, 39011, Spain.

63. Section for Experimental Psychopathology and Neuroimaging, Dept of General Psychiatry, Heidelberg University, Heidelberg, 69120, Germany.

64. Department of Neuroscience, Faculty of Medicine, Norwegian University of Science and Technology (NTNU), Trondheim, 7491, Norway.

65. Department of Radiology, St. Olav's Hospital, Trondheim University Hospital, Trondheim, 7030, Norway.

66. Centre for Academic Mental Health, Population Health Sciences, Bristol Medical School, University of Bristol, Bristol, UK.

67. MRC Integrative Epidemiology Unit, Bristol Medical School, University of Bristol, Oakfield House, Bristol BS8 2BN, UK.

68. NIHR Biomedical Research Centre at the University Hospitals Bristol NHS Foundation Trust and the University of Bristol, Bristol UK.

69. Departments of Radiology and Clinical Neurosciences, University of Calgary, Calgary, Alberta, Canada T2N 4N1.

70. Department of Psychiatry and Mental Health, University of Cape Town, Observatory, Cape Town, 7925, South Africa.

71. South African Medical Research Council (SAMRC) Unit on Risk \& Resilience in Mental Disorders, Cape Town, South Africa. 
72. Laboratory of Epidemiology and Population Sciences, National Institute on Aging, Intramural Research Program, National Institutes of Health, Bethesda, Maryland, 20892, USA.

73. Department of Neurology, Max Planck Institute of Cognitive and Brain Sciences, 04103 Leipzig, Germany.

74. Faculty of Medicine, CRC 1052 Obesity Mechanisms, University of Leipzig, Leipzig, Germany.

75. International Max Planck Research School for Language Sciences, Nijmegen, 6525 XD, The Netherlands.

76. Language and Genetics Department, Max Planck Institute for Psycholinguistics, Nijmegen, 6525 XD, The Netherlands.

77. Department of Psychiatry, University Medicine Greifswald, Greifswald, 17489, Germany.

78. German Center for Neurodegenerative Diseases (DZNE), Rostock/Greifswald, Greifswald, 17487, Germany.

79. Department of Medicine, University of Mississippi Medical Center, Jackson, Mississippi, 39216, USA.

80. Departments of Psychiatry, Neurology, and Psychology, University of Pittsburgh, 3501 Forbes Ave, Suite 830, Pennsylvania 15213, USA.

81. Institute of Molecular Biology and Biochemistry, Centre for Molecular Medicine, Medical University of Graz, Austria.

82. QIMR Berghofer Medical Research Institute, Brisbane, 4006, Australia.

83. Department of Pharmacology, National University of Singapore, Singapore, 119077, Singapore.

84. Memory Aging and Cognition Center, National University Health System, Singapore. 
85. Department of Biomedical Engineering, Illinois Institute of Technology, Chicago, IL 60616, USA.

86. Department of Diagnostic Radiology and Nuclear Medicine, Rush University Medical Center, Chicago, IL, USA.

87. Academic Unit for Psychiatry of Old Age, University of Melbourne, 3101, Australia.

88. National Ageing Research Institute, Royal Melbourne Hospital, Melbourne, 3052, Australia.

89. Harvard Medical School, Boston, Massachusetts, 02115, USA.

90. Lurie Center for Autism, Massachusetts General Hospital, Harvard Medical School, Lexington.

91. Psychiatric and Neurodevelopmental Genetics Unit, Center for Genomic Medicine, Massachusetts General Hospital, Boston, Massachusetts, 02114, USA.

92. Stanley Center for Psychiatric Research, Broad Institute of MIT and Harvard, Boston, Massachusetts, 02141, USA.

93. Department of Psychiatry, University of Oxford, Oxford, OX3 7JX, UK.

94. NIHR Dementia Biomedical Research Unit, King's College London, London, SE5 8AF, UK.

95. NORMENT - KG Jebsen Centre for Psychosis Research, Department of Clinical Science, University of Bergen, 5021, Norway.

96. Dr Einar Martens Research Group for Biological Psychiatry, Center for Medical Genetics and Molecular Medicine, Haukeland University Hospital, Bergen, 5021, Norway.

97. Center for integrated Sequencing, iSEQ, Aarhus University, Aarhus, DK-8000, Denmark.

98. Department of Biomedicine, Aarhus University, Aarhus, DK-8000, Denmark.

99. The Lundbeck Foundation Initiative for Integrative Psychiatric Research, iPSYCH, Aarhus and Copenhagen, DK-8000, Denmark. 
Satizabal et al.

100. Department of Clinical Neuroscience, Centre for Psychiatric Research, Karolinska Institutet, Stockholm, SE-171 77, Sweden.

101. Stockholm Health Care Services, Stockholm County Council, Stockholm, Sweden.

102. UCL Institute of Neurology, London, United Kingdom and Epilepsy Society, Bucks, UK.

103. Centre for Youth Mental Health, The University of Melbourne, Melbourne, VIC, Australia.

104. Orygen, The National Centre of Excellence in Youth Mental Health, Melbourne, Victoria, 3502, Australia.

105. Department of Research and Development, Diakonhjemmet Hospital, Oslo, 0319, Norway.

106. Neuroimaging Unit,Technological Facilities Valdecilla Biomedical Research Institute IDIVAL, Santander, Cantabria, Spain.

107. Avera Institute for Human Genetics, Sioux Falls, South Dakota 57108, USA.

108. Hospital for Sick Children, University of Toronto, Toronto, Ontario, Canada M5G 1X8.

109. Center for Systems Neuroscience, Boston University, Boston, Massachusetts, USA.

110. Institute of Laboratory Medicine, Clinical Chemistry and Molecular Diagnostics, University Hospital Leipzig, 04109 Leipzig, Germany.

111. Groupe d'Imagerie Neurofonctionnelle, Institut des Maladies Neurodégénératives, UMR5293, CEA, CNRS, University of Bordeaux, Bordeaux University Hospital, Bordeaux, France.

112. Pennington Biomedical Research Center, Baton Rouge, LA, 70808.

113. Department of Adult Psychiatry, Institute for Clinical Medicine, University of Oslo, Norway.

114. NORMENT KG Jebsen Centre for Psychosis Research, Oslo University Hospital, Norway.

115. Brain Research Imaging Centre, University of Edinburgh, Edinburgh, EH4 2XU, UK.

116. Department of Computer Science, Lagos State University, Nigeria.

117. Queensland Brain Institute, University of Queensland, Brisbane, 4072, Australia. 
118. Academic Medicine Research Institute, Duke-NUS Graduate Medical School, Singapore.

119. Department of Behavioral Sciences, Rush University Medical Center, Chicago, Illinois, 60612, USA.

120. Centre for Healthy Brain Ageing, School of Psychiatry, University of New South Wales, Sydney, 2052, Australia.

121. Department of Neurology, Johns Hopkins University School of Medicine, Baltimore, MD, 21205, USA.

122. Department of Radiology, Johns Hopkins University School of Medicine, Baltimore, MD, 21205, USA.

123. Department of Psychology, Center for Brain Science, Harvard University, Cambridge, Massachusetts, 02138, USA.

124. Section of Gerontology and Geriatrics, Department of Medicine, University of Perugia, Perugia, 06132, Italy.

125. Center for Multimodal Imaging and Genetics, University of California, San Diego, 92093, California, USA.

126. Departments of Neurosciences, Radiology, Psychiatry, and Cognitive Science, University of California, San Diego, 92093, California, USA.

127. Division of Medical Genetics, Department of Biomedicine, University of Basel, Basel, 4055, Switzerland.

128. Institute of Human Genetics, University of Bonn, Bonn, 53127, Germany.

129. Institute of Neuroscience and Medicine (INM-1), Research Centre Jülich, 52425 Jülich, Germany.

130. Reta Lila Weston Institute and Department of Molecular Neuroscience, UCL Institute of Neurology, London, WC1N 3BG, UK. 
131. Department of ECE, University of New Mexico, Albuquerque, New Mexico, 87131, USA.

132. The Mind Research Network \& LBERI, Albuquerque, New Mexico, 87106, USA.

133. Cerebral Imaging Centre, Douglas Mental Health University Institute, Montreal, QC, Canada.

134. Departments of Psychiatry and Biological and Biomedical Engineering, McGill University, Montreal, QC, Canada.

135. Donders Centre for Cognitive Neuroimaging, Radboud University, Nijmegen, The Netherlands.

136. Institute of Neuroscience and Medicine, Brain \& Behaviour (INM-7), Research Centre Jülich, Jülich, Germany.

137. Department of Clinical Neuropsychology, VU University Amsterdam, Amsterdam, 1081 HV, The Netherlands.

138. Department of Pediatrics, VU Medical Center, Amsterdam, The Netherlands.

139. Emma Children's Hospital Amsterdam Medical Center, Amsterdam, The Netherlands.

140. Univ. Lille, Inserm, Centre Hosp. Univ Lille, Institut Pasteur de Lille, LabEx DISTALZ-

UMR1167 - RID-AGE - Risk factors and molecular determinants of aging-related diseases, Epidemiology and Public Health Department, F-59000 Lille, France.

141. Institute of Diagnostic Radiology and Neuroradiology, University Medicine Greifswald, Greifswald, 17489, Germany.

142. Institute for Translational Genomics and Population Sciences, Los Angeles Biomedical Research Institute and Pediatrics at Harbor-UCLA Medical Center, Torrance, CA 90502.

143. Department of Cognitive Sciences, University of California, San Diego, 92161, USA.

144. Multimodal Imaging Laboratory, Department of Neurosciences, University of California, San Diego, 92093, USA. 
145. Faculty of Health and Institute of Health and Biomedical Innovation, Queensland University of Technology (QUT), Brisbane, 4059, Australia.

146. Dementia Collaborative Research Centre - Assessment and Better Care, UNSW, Sydney, 2052, Australia.

147. 3rd Department of Neurology, Aristotle University of Thessaloniki, Thessaloniki, 'G. Papanicolaou Hospital, 57010, Greece.

148. Division of Mind and Brain Research, Department of Psychiatry and Psychotherapy CCM, Charité - Universitätsmedizin Berlin, corporate member of Freie Universität Berlin, Humboldt-Universität zu Berlin, and Berlin Institute of Health, Berlin, Germany.

149. Brain Center Rudolf Magnus, Human Neurogenetics Unit, UMC Utrecht, Utrecht, 3584 CG, The Netherlands.

150. Department of Molecular and Cellular Therapeutics, the Royal College of Surgeons in Ireland, 123 St Stephen's Green, Dublin 2, Ireland.

151. Department of Psychiatry and Leiden Institute for Brain and Cognition, Leiden University Medical Center, Leiden, 2333 ZA, The Netherlands.

152. Department of Evolution and Genetics, Dagestan State University, Makhachkala, 367000, Dagestan, Russia.

153. Department of Psychiatry, Interdisciplinary Center Psychopathology and Emotion regulation (ICPE), University of Groningen, University Medical Center Groningen, Groningen, The Netherlands.

154. Karakter Child and Adolescent Psychiatry University Center, Nijmegen, The Netherlands.

155. Institute for Community Medicine, Section Epidemiology of Health Care and Community Health, University Medicine Greifswald, Greifswald, Germany. 
156. NORMENT - KG Jebsen Centre, Department of Psychology, University of Oslo, Oslo, 0373, Norway.

157. Centre for Clinical Brain Sciences, University of Edinburgh, Edinburgh, EH16 4SB, UK.

158. Department of Psychiatry and Human Behavior, University of California-Irvine, Irvine, California, 92617, USA.

159. University of Wuerzburg, Department of Psychiatry, Psychosomatics and Psychotherapy, Wuerzburg, Germany.

160. Brain and Mind Centre, University of Sydney, New South Wales, Australia.

161. Neuroscience Research Australia, Sydney, 2031, Australia.

162. University of New South Wales, Australia.

163. Department of Internal Medicine and Geriatric Medicine, INSERM U 558, University of Toulouse, Toulouse, France.

164. Department of Psychiatry and Psychotherapy, Charité Universitätsmedizin Berlin, CCM, Berlin, 10117, Germany.

165. Center for Neurobehavioral Genetics, University of California, Los Angeles, California, 90095, USA.

166. Neurology Division, Beaumont Hospital, Dublin, 9, Ireland.

167. Department of Psychiatry, Carver College of Medicine, University of Iowa, Iowa City, 52242, USA.

168. Interdepartmental Neuroscience Graduate Program, UCLA School of Medicine, Los Angeles, California 90095, USA.

169. Imaging of Dementia and Aging (IDeA) Laboratory, Department of Neurology, University of California-Davis, Davis, CA, 95618, USA. 
Satizabal et al.

170. Department of Epidemiology, Harvard T.H. Chan School of Public Health, Boston, Massachusetts, 02115, USA.

171. KG Jebsen Centre for Psychosis Research / Norwegian Centre for Mental Disorder Research (NORMENT), Institute of Clinical Medicine, University of Oslo, Oslo, Norway.

172. Interfaculty Institute for Genetics and Functional Genomics, University Medicine Greifswald, Greifswald, 17489, Germany.

173. Cardiovascular Health Research Unit, Departments of Medicine, Epidemiology and Health Services, University of Washington, Seattle, Washington, 98101, USA.

174. Kaiser Permanent Washington Health Research Institute, Seattle, WA.

175. Medical and Molecular Genetics, Indiana University School of Medicine, Indianapolis, 46202, USA.

176. Department of Developmental Disability Neuropsychiatry, School of Psychiatry, UNSW Medicine, Australia.

177. Biomedical Research Unit for Dementia, King's College London, London, SE5 8AF, UK.

178. Department of Neuroimaging, Institute of Psychiatry, King's College London, London, SE5 8AF, UK.

179. Division of Clinical Geriatrics, Department of Neurobiology, Care Sciences and Society, Karolinska Institute, Stockholm, Sweden.

180. Division of Genetics, Department of Medicine, Brigham and Women's Hospital, Boston, Massachusetts, 02115, USA.

181. Section of Computational Biomedicine, Department of Medicine, Boston University School of Medicine, Boston, Massachusetts, 02118, USA.

182. Department of Radiology, Mayo Clinic, Rochester, Minnesota, 55905, USA.

183. School of Medical Sciences, UNSW, Sydney, 2052, Australia. 
184. Department of Biomedicine, University of Basel, Basel, Switzerland.

185. Laboratory of Neuro Imaging, USC Mark and Mary Stevens Neuroimaging and Informatics Institute, Keck School of Medicine of the University of Southern California, Los Angeles, California, 90033, USA.

186. Translational Genomics Research Institute, Neurogenomics Division, 445 N Fifth Street, Phoenix, Arizona 85004, USA.

187. Radiation Sciences, Umeå University, S-901 87 Umeå, Sweden.

188. Department of Radiology, Massachusetts General Hospital, Harvard Medical School, Boston, Massachusetts, USA.

189. Departments of Psychiatry, Neurology, Neuroscience and the Institute of Genetic Medicine, Johns Hopkins University School of Medicine, Baltimore, 21205, USA.

190. Biological Psychology, Vrije Universiteit Amsterdam, Amsterdam, The Netherlands.

191. Imaging Physics, Faculty of Applied Sciences, Delft University of Technology, The Netherlands.

192. Department of Medical Informatics, Erasmus MC, Rotterdam, 3015 CE, The Netherlands.

193. Institut Pasteur, Paris, 75015, France.

194. Departments of Neurology and Epidemiology, University of Washington, Seattle, WA, USA, 325 Ninth Avenue, Seattle WA, 98104-2420, USA.

195. Neuroimaging Centre, University of Groningen, University Medical Center Groningen, Groningen, 9713 AW, The Netherlands.

196. Rotman Research Institute, University of Toronto, Toronto, Ontario, Canada M6A 2 E1.

197. INSERM UMR 1000 “Neuroimaging and Psychiatry”, University Paris-Sud, University ParisSaclay, University Paris Descartes; Service Hospitalier Frédéric Joliot, Orsay; and Maison de Solenn, Paris; France. 
Satizabal et al.

198. HMNC Brain Health, Munich, 80539, Germany.

199. Department of Neurology, Hopital Erasme, Universite Libre de Bruxelles, Brussels, 1070, Belgium.

200. Department of Psychology, Georgia State University, Atlanta, GA, USA.

201. Department of Psychiatry, University of Groningen, University Medical Center Groningen, Groningen, The Netherlands.

202. Department of Medical Genetics, Oslo University Hospital, Oslo, 0450, Norway.

203. Centre for Advanced Imaging, University of Queensland, Brisbane, 4072, Australia.

204. Munich Cluster for Systems Neurology (SyNergy), Munich, 81377, Germany.

205. University of Liverpool, Institute of Translational Medicine, Liverpool, L69 3BX, UK.

206. Institute of Clinical Medicine, Neurology, University of Eastern Finland, Kuopio, FI-70211, Finland.

207. Neurocentre Neurology, Kuopio University Hospital, FI-70211, Finland.

208. Institute of Clinical Chemistry and Laboratory Medicine, University Medicine Greifswald, Greifswald, 17489, Germany.

209. German Center for Cardiovascular Research (DZHK eV), partner site Greifswald, Germany.

210. Department of Neuroinformatics, Araya, Inc., Tokyo, 105-0001, Japan.

211. Institute of Cognitive Neuroscience, University College London, London, WC1N 3AR, UK.

212. School of Psychology, University of Sussex, Brighton, BN1 9QH, UK.

213. Department of Neurobiology, Care Sciences and Society, Karolinska Institutet, Stockholm, SE-141 83, Sweden.

214. Department of Child and Adolescent Psychiatry/Psychology, Erasmus MC-Sophia Children's Hospital, Rotterdam, 3015 CE, The Netherlands. 
Satizabal et al.

215. Department of Clinical Genetics, Maastricht University Medical Center, Maastricht, 6200 MD, The Netherlands.

216. GROW School for Oncology and Developmental Biology, Maastricht, The Netherlands.

217. Department of Genomics, Life \& Brain Center, University of Bonn, 53127, Germany.

218. Neuropsychiatric Institute, Prince of Wales Hospital, Sydney, 2031, Australia.

219. Department of Psychology, VU University Amsterdam, Amsterdam, 1081 BT, The Netherlands.

220. Center for Imaging of Neurodegenerative Disease, San Francisco VA Medical Center, University of California, San Francisco, 94121, USA.

221. Department of Radiology and Biomedical Imaging, University of California, San Francisco, California, 94143, USA.

222. Leiden Academic Centre for Drug Research (LACDR), Leiden University, The Netherlands.

223. Departments of Neurology and Psychiatry, University of Pittsburgh, 3501 Forbes Ave, Suite 830 Pittsburgh PA 15213, USA.

224. Institute of Molecular Medicine and Human Genetics Center, University of Texas Health Science Center at Houston, Houston, Texas, 77030, USA.

225. UK Dementia Research Institute at the University of Edinburgh, Chancellor's Building, Little France, Edinburgh H16 4SB.

226. Department of Neurology and Center for Neuroscience, University of California at Davis, Sacramento, California, 95817, USA.

227. Center for Translational \& Computational Neuroimmunology, Department of Neurology, Columbia University Medical Center, New York, NY 10032, USA.

228. Departments of Neurology, Molecular \& Human Genetics, Neuroscience, and Program in Developmental Biology, Baylor College of Medicine, Houston, Texas, 77030, USA. 
bioRxiv preprint doi: https://doi.org/10.1101/173831; this version posted August 28,2017 . The copyright holder for this preprint (which was not certified by peer review) is the author/funder. All rights reserved. No reuse allowed without permission.

Satizabal et al.

229. Jan and Dan Duncan Neurological Research Institute, Texas Children's Hospital, Houston, TX, USA. 
Abstract: 123

Text: 3,092

Tables: 1

Figures: 3

References: 76

Supplementary tables: 18

Correspondence to:

\section{Dr. Claudia L. Satizabal}

Boston University School of Medicine and The Framingham Heart Study

72 East Concord Street, B-601, Boston, MA 02118-2526, USA

Phone: +1 617-638-5398

Email: clausati@bu.edu

Or,

\section{Dr. M. Arfan Ikram}

Erasmus University Medical Center Rotterdam

P.0. Box 2040, 3000 CA, Rotterdam, The Netherlands

Phone: +31 107043930

E-mail: m.a.ikram@erasmusmc.nl 
Satizabal et al.

\begin{abstract}
Subcortical brain structures are integral to motion, consciousness, emotions, and learning. We identified common genetic variation related to the volumes of nucleus accumbens, amygdala, brainstem, caudate nucleus, globus pallidus, putamen, and thalamus, using genome-wide association analyses in over 40,000 individuals from CHARGE, ENIGMA and the UK-Biobank. We show that variability in subcortical volumes is heritable, and identify 25 significantly associated loci (20 novel). Annotation of these loci utilizing gene expression, methylation, and neuropathological data identified 62 candidate genes implicated in neurodevelopment, synaptic signaling, axonal transport, apoptosis, and susceptibility to neurological disorders. This set of genes is significantly enriched for Drosophila orthologs associated with neurodevelopmental phenotypes, suggesting evolutionarily conserved mechanisms. Our findings uncover novel biology and potential drug targets underlying brain development and disease.
\end{abstract}


Satizabal et al.

Subcortical brain structures are essential for the control of autonomic and sensorimotor functions $s^{1,2}$, modulation of processes involved in learning, memory, and decision-making 3,4 , as well as in emotional reactivity 5,6 and consciousness ${ }^{7}$. They often act through networks influencing input to and output from the cerebral cortex ${ }^{8,9}$. The pathology of many cognitive, psychiatric, and movement disorders is restricted to, begins in, or predominantly involves subcortical brain structures and related circuitries ${ }^{10}$. For instance, tau pathology has shown to manifest itself early in the brainstem and thalamic nuclei of individuals with Alzheimer's disease before spreading to cortical areas through efferent networks ${ }^{11}$. Similarly, the formation of Lewy bodies and Lewy neurites in Parkinson's disease appears early in the lower brainstem (and olfactory structures) before affecting the substantia nigra ${ }^{12}$.

A recent investigation identified five novel genetic loci influencing the volumes of the putamen and caudate, which pointed to genes controlling neuronal growth, apoptosis, and learning13. However, no genome-wide significant signals associated with the volumes of the nucleus accumbens, amygdala, globus pallidus, and thalamus were detected, and the genetic variation associated with brainstem volume has not been previously explored. Identifying novel genetic factors contributing to variability in subcortical structures, including the brainstem, should further improve our understanding of brain development and disease.

We sought to identify novel genetic variants influencing the volumes of seven subcortical structures (nucleus accumbens, amygdala, caudate nucleus, putamen, globus pallidus, thalamus, and brainstem (including mesencephalon, pons, and medulla oblongata)), through genome-wide association (GWA) analyses in over 40,000 individuals from 54 study samples (Table S1) from the Cohorts of Heart and Aging Research in Genomic Epidemiology (CHARGE) consortium, the Enhancing Neuro Imaging Genetics through Meta-Analysis (ENIGMA) consortium, and the United Kingdom Biobank (UKBB). 
Satizabal et al.

\section{RESULTS}

\section{Heritability}

To examine the extent to which genetic variation accounts for variation in subcortical brain volumes, we estimated the heritability of those volumes in the Framingham Heart Study (FHS) and the Austrian Stroke Prevention Study (ASPS-Fam) family-based cohorts. Our analyses are in line with previous studies conducted in young ${ }^{14}$ and older ${ }^{15}$ twins, suggesting that variability in subcortical volumes is moderately to highly heritable. The structures with highest heritability in the FHS and the ASPS-Fam family-based cohorts are the brainstem (ranging from 79-86\%), caudate nucleus (71-85\%), putamen (71-79\%) and nucleus accumbens (66\%); followed by the globus pallidus (55-60\%), thalamus (47-54\%), and amygdala (34-59\%) (Figure 1 and Supplementary Table S2).

\section{Genome-wide associations}

We undertook a GWA analysis on the MRI-derived volumes of subcortical structures using the 1000 Genomes Project1516 reference panel (phase 1 v.3) for imputation of missing variants. Our discovery sample comprised up to $n=25,587$ individuals of European ancestry from 45 study samples in CHARGE and ENIGMA (Table S1). Additionally, we included four samples for replication in Europeans (up to $n=13,707$ ), three for generalization to African-Americans (up to $n=769$ ), and two for generalization to Asians $(n=341)$. Each study related genetic variants with minor allele frequency (MAF) $\geq 1 \%$ to the volumes of subcortical structures (average volume for bilateral structures) using additive genetic models adjusted for sex, age, age ${ }^{2}$, total intracranial volume (total brain volume in the UKBB), and population structure. After quality control, we combined studyspecific GWA results using sample-size-weighted fixed effects methods in METAL ${ }^{16}$. We conducted 
Satizabal et al.

meta-analyses in stages, from discovery, through replication and generalization, to the combination of all available samples.

In the discovery analysis, we identified 25 genome-wide significant loci across six subcortical structures, 20 of which are novel (Table 1). Among them, 13 variants were located within genes (one 3'-UTR, one missense, one non-coding transcript, 10 intronic), and 12 in intergenic regions. In addition to these 25 loci, a further seven novel probable genetic associations were identified: four had p-values just above the threshold of significance $\left(5.3 \times 10^{-8}\right.$ to $\left.2.9 \times 10^{-7}\right)$ and three others reached genome-wide significance but were less frequent variants reliably genotyped in a smaller sample of $\mathrm{n}<2500$ individuals. Replication results in the UKBB are shown in Table 1 . We carried forward these 32 loci pointing to 31 candidate genes (variants at the 14q22.3 locus near KTN1 were related to putamen and globus pallidus volumes) to in-silico replication in Europeans, generalization in African-Americans and Asians, and combined meta-analysis of all samples (Table S3). Of 32 candidate loci, the direction of association was the same for 24 variants in Europeans and 15 variants across all ethnicities. In the combined meta-analysis, 21 of the 32 associations were genome-wide significant, 20 for which the strength of association increased from the discovery. Among these, are 2 of variants for the nucleus accumbens (MAST4 and SNAR-I) below the threshold in the discovery now reached genome-wide significance in the combined meta-analysis.

To functionally annotate our discoveries, we investigated expression quantitative trait loci (eQTL, Table S4) and methylation QTL (meQTL, Table S5) for the 32 candidate loci identified in the discovery analysis, using data from post-mortem brains from the Religious Order Study and the Rush Memory and Aging Project (ROSMAP). We also queried a variety of cis- and trans-eQTL datasets in brain and non-brain tissues (further described in the Supplement) for the 32 candidate loci or their proxies $\left(r^{2}>0.8\right)$, using the European population reference (Table S6). This allowed us to identify 31 additional candidate genes (in addition to the 31 candidate genes within or near the 
Satizabal et al.

32 loci carried forward for in-silico replication), including one long intergenic non-protein coding RNA, and one microRNA, yielding a final set of 62 candidate genes (Table S7). The details describing the process, whereby specific genes were identified at each locus, can be found in the supplement (see extended results in the Supplementary note).

\section{Associations with cognitive function and neuropathological phenotypes}

We related genetic variation of the 32 variants as well as the expression of our final set of 62 genes influencing subcortical brain volumes to cognitive function and neuro-pathological traits in ROSMAP. We did not find significant associations for individual variants with any investigated trait after Bonferroni correction $(\mathrm{P}<0.0003)$, except for the $A P O E$ variant rs429358, which was, not surprisingly, associated with the presence of neurofibrillary tangles, tau density, $\beta$-amyloid load, neuritic plaques, and cognitive decline (Table S8). However, we did find significant associations of dorsolateral prefrontal cortex mRNA expression levels of five candidate genes influencing brainstem, caudate, and putamen volumes (Table S9). These included associations with cognitive function (KTN1, BCL2L1, SGTB, C20orf166-AS1, PTCH1), neuritic plaque presence (BCL2L1, KTN1), $\beta$-amyloid load (SGTB, KTN1), neurofibrillary tangles (BCL2L1), and tau density (BCL2L1).

\section{Phenotypic and genetic correlations}

We explored both phenotypic and genetic correlations among subcortical volumes, and also the genetic correlations between subcortical volumes and height, MRI-defined hippocampal17 and intracranial18 volumes, adult height ${ }^{19}$, body mass index ${ }^{20}$, Alzheimer's disease ${ }^{21}$, general cognitive function $^{22}$, bipolar disorder ${ }^{23}$, and schizophrenia ${ }^{24}$; using linkage disequilibrium (LD) score regression methods ${ }^{25}$ (Figure 2 and Supplementary Table S10). We observed strong phenotypic $(\mathrm{P}<3.95 \mathrm{E}-06)$ and genetic $\left(\mathrm{P}=0.04-4.5 \times 10^{-17}\right)$ overlap among all subcortical structures (Figure $\left.2 \mathrm{~A}\right)$, 
Satizabal et al.

consistent with our finding that many of the loci identified have pleiotropic effects on the volumes of several subcortical structures (Table S3).

As expected, we found strong genetic correlations among the nuclei composing the corpus striatum, particularly for nucleus accumbens with putamen $\left(\mathrm{P}=1.24 \times 10^{-14}\right)$, and with caudate nucleus $\left(\mathrm{P}=6.92 \times 10^{-13}\right)$. The genetic architecture of thalamic volume highly overlapped with that of most subcortical volumes, except for the nucleus accumbens. In contrast, there were no significant genetic correlation of the volume of the brainstem with that of most other structures, with the exception of very strong correlations with volumes of the thalamus $\left(\mathrm{P}=4.45 \times 10^{-17}\right)$ and the globus pallidus $\left(\mathrm{P}=9.20 \times 10^{-09}\right)$.

We also observed strong genetic correlations of smaller amygdala and putamen volumes with increased risk of Alzheimer's disease, and smaller nucleus accumbens and caudate nucleus volumes with risk of bipolar disorder. Increased general cognitive function was correlated with larger brainstem, thalamic, and nucleus accumbens volumes. Finally, intracranial volume was genetically correlated with larger volumes of subcortical structures, except for the nucleus accumbens and the putamen (Figure 2B).

\section{Cross-species analysis}

To investigate for potential evolutionarily conserved requirements of our gene-set in neurodevelopment, neuronal maintenance, or both, we examined available genetic and phenotypic data from the fruit fly, Drosophila melanogaster. Importantly, compared to mammalian models, the fly genome has been more comprehensively interrogated for roles in the nervous system. We found that the majority of candidate genes for human subcortical volumes are strongly conserved in the Drosophila genome (66.1\%), and many of these genes appear to have conserved nervous system requirements (Table S11). To examine if this degree of conservation was greater than that expected 
Satizabal et al.

by chance, we leveraged systematic, standardized phenotype data based on FlyBase annotations using controlled vocabulary terms (Table S12). Indeed, $24.1 \%$ of the conserved fly homologs are documented to cause "neuroanatomy defective" phenotypes in flies, representing a significant $\left(\mathrm{P}=3.9 \times 10^{-3}\right)$, nearly two-fold enrichment compared to $12.9 \%$ representing all Drosophila genes associated with such phenotypes (Table S13).

\section{Protein-protein interactions}

To explore potential functional relationships between proteins encoded by our set of 62 genes, we conducted protein-protein interaction analyses in STRING ${ }^{26}$. Our results revealed enrichment of genes involved in brain-specific pathways (i.e. nervous system development, regulation of neuronal death, neuron projection, axon, neuron part), as well as housekeeping processes (i.e. cell differentiation, apoptosis, kinase binding). Figure 3 shows these protein networks, and the detailed pathways are presented in Table S14. 
Satizabal et al.

\section{DISCUSSION}

We undertook the largest GWA meta-analysis of variants associated with MRI-derived volumes of the nucleus accumbens, amygdala, brainstem, caudate nucleus, globus pallidus, putamen, and thalamus; in more than 40,000 individuals from 54 study samples worldwide. Our analyses identified a set of 62 candidate genes influencing the volume of these subcortical brain structures, most of which have well-established roles in the nervous system.

We identified genes implicated in neurodevelopmental processes, including all the candidates influencing the volume of the caudate nucleus. We confirm one locus in 11q14.3 near the FAT3 gene previously associated with the caudate nucleus ${ }^{13}$, where the top variant is an eQTL for the expression of FAT3 in CD14+ monocytes (Table S6). This gene encodes a conserved cellular adhesion molecule implicated in neuronal morphogenesis and cell migration based on mouse genetic studies ${ }^{27}$. Variants in a locus on $9 q 33$ located $150 \mathrm{~kb}$ from $P B X 3$ were also significantly associated with caudate volume. $P B X 3$ is robustly expressed in the developing caudate nucleus of the non-human primate, Macaca fuscata, consistent with a role in striatal neurogenesis ${ }^{28}$. Another locus associated with caudate volume at $2 \mathrm{p} 21$ is $40 \mathrm{~kb}$ proximal to SIX3, which encodes a transcriptional regulator with conserved neurodevelopmental roles in both vertebrates and invertebrates ${ }^{29}$.The most significant variant at this locus is associated with $\mathrm{CpG}$ sites near active transcription start sites (TSS) harboring SIX3 in anterior caudate brain tissues (Figure S3.F). Finally, another locus associated with caudate volume was at the $9 \mathrm{q} 22.3$ locus, $97 \mathrm{~kb}$ upstream of PTCH1, encoding a receptor for the Sonic Hedgehog (SHH) signaling protein, which was also recently found associated with hippocampal volume17. Mutations in PTCH1 and SHH are responsible for a third of medulloblastomas ${ }^{30}$. In addition, dominant mutations in SIX3, PTCH1, and SHH similarly cause human holoprosencephaly ${ }^{31}$, and their genetic manipulation causes analogous developmental 
Satizabal et al.

phenotypes in mice ${ }^{30,32}$. Moreover, $S H H$ is a direct transcriptional target of $S I X 3^{33}$, raising the possibility that this pathway also regulates caudate development.

Furthermore, in our GWA of brainstem volume we identified a signal at $4 \mathrm{q} 22,185 \mathrm{~kb}$ downstream of ATOH1, an important gene for neurodevelopment. ATOH1 encodes an evolutionarily conserved transcriptional regulator of neuronal differentiation, based on studies in numerous animal models ${ }^{34}$. Mice lacking Math1, the ATOH1ortholog, show widespread brainstem developmental anomalies ${ }^{35}$, including disruption of medullary and pontine nuclei with roles in respiratory drive ${ }^{36}$. The most significant variant in this locus is also an eQTL for the expression of SMARCAD1 and GRID2 in blood cells (Table S6). In mouse experimental models, expression of Smarcad1 accompanies neurogenesis ${ }^{37}$; whereas in Lurcher mice, serving as a model for neurodegeneration, mutations in Grid2 are characterized by brainstem and cerebellar neurodegeneration ${ }^{38}$ resulting in ataxia ${ }^{39}$. We found that variants in PAPPA and IGF1 are associated with the volumes of the brainstem and caudate nucleus, respectively. PAPPA encodes a secreted metalloproteinase that cleaves IGFBPs, thereby releasing bound IGF. Although IGF may be beneficial in early- and midlife (i.e. higher levels are associated with larger brain volumes and a lower risk of Alzheimer's disease ${ }^{40}$ ); its effects may be detrimental during aging, and studies of PAPPA similarly support antagonistic pleiotropy. Low circulating PAPPA levels are a marker for adverse outcomes in human embryonic development ${ }^{41}$, but in later life, higher levels have been associated with acute coronary syndromes and heart failure ${ }^{42,43}$. Similarly, Pappa knockout mice show dwarfism but reduced age-related degeneration and increased longevity 44 .

In screening for variants associated with globus pallidus volume, we identified additional genes involved in neurodevelopment. One was an intronic variant in $A L P L$, associated with CpG sites near enhancers in the gene and transcription sites in NBPF3 (Table S5 and Figure S3.I). ALPL encodes an alkaline phosphatase that mediates bone mineralization, regulates cell migration, neuronal 
Satizabal et al.

differentiation early during development, and post-natal synaptogenesis in transgenic mouse models ${ }^{45}$. Recent reports suggest that ALPL helps propagate the neurotoxicity induced by tau ${ }^{46}$, and its activity increases in Alzheimer's disease $\mathrm{e}^{47}$ and cognitive impairment ${ }^{48}$. NBPF3 belongs to the neuroblastoma breakpoint family, which encodes domains of the autism- and schizophrenia-related DUF1220 protein 49 .

Genes influencing the volume of the thalamus, a relay hub for electrical impulses travelling between subcortical structures and the cerebral cortex, were related to synaptic signaling pathways. We found a missense variant in NPTX1, a gene expressed in the nervous system which restricts synapse plasticity50, and induces $\beta$-amyloid neurodegeneration in human and mouse brain tissues ${ }^{51}$. We also identified an intronic variant in NCAM2, encoding a protein involved in olfactory system development ${ }^{52}$, levels of which are lower in hippocampal synapses of Alzheimer's disease brains ${ }^{53}$, possibly contributing to synapse loss in Alzheimer's disease.

Additionally, the identified variant at the 3'-UTR of SGTB for the brainstem was a robust eQTL for the expression of SGTB in cerebellum, visual cortex (Table S6), and dorsolateral prefrontal cortex (Table S4). Experimental rat models showed that $\beta$ SGT, highly expressed in brain, forms a complex with the cysteine string protein and heat-shock protein cognate (CSP/Hsc70) complex to function as a chaperone guiding the refolding of misfolded proteins near synaptic vesicles ${ }^{54}$. Other experimental studies in the nematode worm, C. elegans, showed that the genetic manipulation of the ortholog, sgt-1, suppresses toxicity associated with expression of the human $\beta$-amyloid peptide ${ }^{55}$. Other genes involved in synaptic signaling are CHPT1 (brainstem), involved in phosphatidylcholine metabolism in the brain, and DLG2 (putamen), encoding an evolutionarily conserved scaffolding protein involved in glutamatergic-mediated synaptic signaling and cell 
Satizabal et al.

polarity ${ }^{56}$ that has been associated with schizophrenia ${ }^{57}$, cognitive impairment ${ }^{58}$, and Parkinson's disease ${ }^{59}$.

Other identified variants point to genes involved in autophagy and apoptotic processes, such as DRAM1 and FOXO3, both related to brainstem volumes. DRAM1 encodes a lysosomal membrane protein involved in activating TP53-mediated autophagy and apoptosis, ${ }^{60}$ and mouse models mimicking cerebral ischemia and reperfusion have found that inhibiting the expression of DRAM1 worsens cell injury ${ }^{61}$. The most significant variant located $9 \mathrm{~Kb}$ downstream from DRAM1 was also associated with a CpG site proximate to active TSS upstream of that gene in several mature brain tissues (Table S5 and Figure S3.B). FOXO3 has been recently identified as pivotal in an astrocyte network conserved across humans and mice involved in stress, sleep, and Huntington's disease ${ }^{62}$, and has been related to longevity ${ }^{63}$. In Drosophila, a FOXO3 ortholog regulates dendrite number and length in the peripheral nervous system ${ }^{64}$, and in the zebrafish, Danio rario, Foxo3a knockdown led to apoptosis and mispatterning of the embryonic CNS65.

Finally, some of the genes we identified have been implicated in axonal transport. Our results confirm an association between variants in the $13 q 22$ locus with putamen and globus pallidus volumes as previously reported ${ }^{13,66}$. The most significant variant (rs8017172) is a robust eQTL for KTN1 in peripheral blood cells (Table S6). This gene encodes a kinesin-binding protein involved in the transport of cellular components along microtubules ${ }^{67}$, and impairment of these molecular motors has been increasingly recognized in neurological diseases with a subcortical component ${ }^{68}$. The $5 q 12$ locus, associated with nucleus accumbens volume in the combined analysis, lies $53 \mathrm{~kb}$ upstream from MAST4, which encodes a member of the microtubule-associated serine/threonine kinases. This gene has been associated with hippocampal volumes ${ }^{17}$ and juvenile myoclonic 
Satizabal et al.

epilepsy ${ }^{69}$, and it appears to be differentially expressed in the prefrontal cortex of atypical cases of frontotemporal lobar degeneration ${ }^{70}$. In Drosophila, the knockdown of a conserved MAST4 homolog enhanced the neurotoxicity of human tau 71 , which aggregates to form neurofibrillary tangle pathology in Alzheimer's disease.

Overall, the loci identified by our study pinpoint candidate genes not only associated with human subcortical brain volumes, but also reported to disrupt invertebrate neuroanatomy when manipulated in Drosophila and many other animal models. This is consistent with the results observed in protein-protein networks. Thus, our results are in line with the knowledge that the genomic architecture of central nervous system development has been strongly conserved during evolution. Further elaboration of the biological pathways associated with the genes not discussed in the main text may be found in the Supplementary note (see extended results).

Our findings derived from genetic correlations support earlier observations that amygdala volume is reduced in Alzheimer's disease patients ${ }^{72}$ and in carriers of the Alzheimer risk enhancing $\varepsilon 4$ variant of the APOE gene ${ }^{73}$. Interestingly, one of the top signals related to the amygdala was one of the two variants that determines the APOE $\varepsilon 4$ isoform (rs429358). In line with our findings, other studies have described smaller putamen volumes in Alzheimer's disease ${ }^{74}$, or smaller accumbens and caudate nuclei in patients with bipolar disorder ${ }^{75,76}$. Notably, higher general cognitive function was correlated with larger brainstem, thalamus, and nucleus accumbens, highlighting the integrative role of these brain structures in cognition. 
In conclusion, we describe multiple genes associated with the volumes of MRI-derived subcortical structures in a large sample, leveraging diverse bioinformatic resources to validation and follow-up our findings. Our analyses indicate that the variability of evolutionarily old subcortical volumes of humans is moderately to strongly heritable, and that their genetic variation is also strongly conserved across different species. The majority of the variants identified in this analysis point to genes involved in neurodevelopment, regulation of neuronal apoptotic processes, synaptic signaling, brain homeostasis, and susceptibility to neurological disorders. We show that the genetic architecture of subcortical volumes overlaps with that of anthropometric measures and neuropsychiatric disorders. We have focused on the discovery of common and less frequent variants, but further efforts to also reveal rare variants and epigenetic signatures associated with subcortical structures will provide an even more refined understanding of the underlying mechanisms involved. In summary, our findings greatly expand current understanding of the genetic variation related to subcortical structures, which can help identify novel biological pathways of relevance to human brain development and disease. 
Satizabal et al.

\section{REFERENCES}

1 Marsden, C. D. The mysterious motor function of the basal ganglia: the Robert Wartenberg Lecture. Neurology 32, 514-539 (1982).

2 Yin, H. H. \& Knowlton, B. J. The role of the basal ganglia in habit formation. Nature reviews. Neuroscience 7, 464-476, doi:10.1038/nrn1919 (2006).

3 McDonald, A. J. \& Mott, D. D. Functional neuroanatomy of amygdalohippocampal interconnections and their role in learning and memory. Journal of neuroscience research, doi:10.1002/jnr.23709 (2016).

4 Hikosaka, O., Kim, H. F., Yasuda, M. \& Yamamoto, S. Basal ganglia circuits for reward valueguided behavior. Annual review of neuroscience 37, 289-306, doi:10.1146/annurev-neuro071013-013924 (2014).

5 Salzman, C. D. \& Fusi, S. Emotion, cognition, and mental state representation in amygdala and prefrontal cortex. Annual review of neuroscience 33, 173-202, doi:10.1146/annurev.neuro.051508.135256 (2010).

6 Floresco, S. B. The nucleus accumbens: an interface between cognition, emotion, and action. Annual review of psychology 66, 25-52, doi:10.1146/annurev-psych-010213-115159 (2015).

7 Fabbro, F., Aglioti, S. M., Bergamasco, M., Clarici, A. \& Panksepp, J. Evolutionary aspects of self- and world consciousness in vertebrates. Frontiers in human neuroscience $\mathbf{9}, 157$, doi:10.3389/fnhum.2015.00157 (2015).

8 Alexander, G. E., DeLong, M. R. \& Strick, P. L. Parallel organization of functionally segregated circuits linking basal ganglia and cortex. Annual review of neuroscience 9, 357-381, doi:10.1146/annurev.ne.09.030186.002041 (1986). 
Satizabal et al.

9 Jahanshahi, M., Obeso, I., Rothwell, J. C. \& Obeso, J. A. A fronto-striato-subthalamic-pallidal network for goal-directed and habitual inhibition. Nature reviews. Neuroscience 16, 719732, doi:10.1038/nrn4038 (2015).

10 Shepherd, G. M. Corticostriatal connectivity and its role in disease. Nature reviews. Neuroscience 14, 278-291, doi:10.1038/nrn3469 (2013).

11 Stratmann, K. et al. Precortical Phase of Alzheimer's Disease (AD)-Related Tau Cytoskeletal Pathology. Brain pathology 26, 371-386, doi:10.1111/bpa.12289 (2016).

12 Del Tredici, K., Rub, U., De Vos, R. A., Bohl, J. R. \& Braak, H. Where does Parkinson disease pathology begin in the brain? Journal of neuropathology and experimental neurology $\mathbf{6 1}$, 413-426 (2002).

13 Hibar, D. P. et al. Common genetic variants influence human subcortical brain structures. Nature 520, 224-229, doi:10.1038/nature14101 (2015).

14 Renteria, M. E. et al. Genetic architecture of subcortical brain regions: common and regionspecific genetic contributions. Genes, brain, and behavior 13, 821-830, doi:10.1111/gbb.12177 (2014).

15 Clarke, L. et al. The 1000 Genomes Project: data management and community access. Nature methods 9, 459-462, doi:10.1038/nmeth.1974 (2012).

16 Willer, C. J., Li, Y. \& Abecasis, G. R. METAL: fast and efficient meta-analysis of genomewide association scans. Bioinformatics 26, 2190-2191, doi:10.1093/bioinformatics/btq340 (2010).

17 Hibar, D. P. et al. Novel genetic loci associated with hippocampal volume. Nature communications $\mathbf{8}, 13624$, doi:10.1038/ncomms13624 (2017). 
Satizabal et al.

18 Adams, H. H. et al. Novel genetic loci underlying human intracranial volume identified through genome-wide association. Nature neuroscience 19, 1569-1582, doi:10.1038/nn.4398 (2016).

19 Wood, A. R. et al. Defining the role of common variation in the genomic and biological architecture of adult human height. Nature genetics 46, 1173-1186, doi:10.1038/ng.3097 (2014).

20 Locke, A. E. et al. Genetic studies of body mass index yield new insights for obesity biology. Nature 518, 197-206, doi:10.1038/nature14177 (2015).

21 Lambert, J. C. et al. Meta-analysis of 74,046 individuals identifies 11 new susceptibility loci for Alzheimer's disease. Nature genetics 45, 1452-1458, doi:10.1038/ng.2802 (2013).

22 Davies, G. et al. Genetic contributions to variation in general cognitive function: a metaanalysis of genome-wide association studies in the CHARGE consortium ( $\mathrm{N}=53949)$. Molecular psychiatry 20, 183-192, doi:10.1038/mp.2014.188 (2015).

23 Psychiatric, G. C. B. D. W. G. Large-scale genome-wide association analysis of bipolar disorder identifies a new susceptibility locus near ODZ4. Nature genetics 43, 977-983, doi:10.1038/ng.943 (2011).

24 Schizophrenia Working Group of the Psychiatric Genomics, C. Biological insights from 108 schizophrenia-associated genetic loci. Nature 511, 421-427, doi:10.1038/nature13595 (2014).

25 Bulik-Sullivan, B. et al. An atlas of genetic correlations across human diseases and traits. Nature genetics 47, 1236-1241, doi:10.1038/ng.3406 (2015).

26 Szklarczyk, D. et al. STRING v10: protein-protein interaction networks, integrated over the tree of life. Nucleic acids research 43, D447-452, doi:10.1093/nar/gku1003 (2015). 
Satizabal et al.

27 Deans, M. R. et al. Control of neuronal morphology by the atypical cadherin Fat3. Neuron 71, 820-832, doi:10.1016/j.neuron.2011.06.026 (2011).

28 Takahashi, K. et al. Expression of FOXP2 in the developing monkey forebrain: comparison with the expression of the genes FOXP1, PBX3, and MEIS2. The Journal of comparative neurology 509, 180-189, doi:10.1002/cne.21740 (2008).

29 Kumar, J. P. The sine oculis homeobox (SIX) family of transcription factors as regulators of development and disease. Cellular and molecular life sciences : CMLS 66, 565-583, doi:10.1007/s00018-008-8335-4 (2009).

30 Skowron, P., Ramaswamy, V. \& Taylor, M. D. Genetic and molecular alterations across medulloblastoma subgroups. Journal of molecular medicine $\mathbf{9 3 , 1 0 7 5 - 1 0 8 4 ,}$ doi:10.1007/s00109-015-1333-8 (2015).

31 Cohen, M. M., Jr. Holoprosencephaly: clinical, anatomic, and molecular dimensions. Birth defects research. Part A, Clinical and molecular teratology 76, 658-673, doi:10.1002/bdra.20295 (2006).

32 Aoto, K. \& Trainor, P. A. Co-ordinated brain and craniofacial development depend upon Patched1/XIAP regulation of cell survival. Human molecular genetics 24, 698-713, doi:10.1093/hmg/ddu489 (2015).

33 Geng, X. et al. Haploinsufficiency of Six3 fails to activate Sonic hedgehog expression in the ventral forebrain and causes holoprosencephaly. Developmental cell 15, 236-247, doi:10.1016/j.devcel.2008.07.003 (2008).

34 Huang, C., Chan, J. A. \& Schuurmans, C. Proneural bHLH genes in development and disease. Current topics in developmental biology 110, 75-127, doi:10.1016/B978-0-12-4059436.00002-6 (2014). 
Satizabal et al.

35 Wang, V. Y., Rose, M. F. \& Zoghbi, H. Y. Math1 expression redefines the rhombic lip derivatives and reveals novel lineages within the brainstem and cerebellum. Neuron 48, 3143, doi:10.1016/j.neuron.2005.08.024 (2005).

36 Rose, M. F. et al. Math1 is essential for the development of hindbrain neurons critical for perinatal breathing. Neuron 64, 341-354, doi:10.1016/j.neuron.2009.10.023 (2009).

37 Lim, D. A. et al. In vivo transcriptional profile analysis reveals RNA splicing and chromatin remodeling as prominent processes for adult neurogenesis. Molecular and cellular neurosciences 31, 131-148, doi:10.1016/j.mcn.2005.10.005 (2006).

38 Cheng, S. S. \& Heintz, N. Massive loss of mid- and hindbrain neurons during embryonic development of homozygous lurcher mice. The Journal of neuroscience : the official journal of the Society for Neuroscience 17, 2400-2407 (1997).

39 Lalouette, A., Guenet, J. L. \& Vriz, S. Hotfoot mouse mutations affect the delta 2 glutamate receptor gene and are allelic to lurcher. Genomics 50, 9-13, doi:10.1006/geno.1998.5314 (1998).

40 Westwood, A. J. et al. Insulin-like growth factor-1 and risk of Alzheimer dementia and brain atrophy. Neurology 82, 1613-1619, doi:10.1212/WNL.0000000000000382 (2014).

41 Kjaer-Sorensen, K. et al. Pregnancy-associated plasma protein A (PAPP-A) modulates the early developmental rate in zebrafish independently of its proteolytic activity. The Journal of biological chemistry 288, 9982-9992, doi:10.1074/jbc.M112.426304 (2013).

42 Bayes-Genis, A. et al. Pregnancy-associated plasma protein A as a marker of acute coronary syndromes. The New England journal of medicine 345, 1022-1029, doi:10.1056/NEJMoa003147 (2001).

43 Funayama, A. et al. Serum pregnancy-associated plasma protein a in patients with heart failure. Journal of cardiac failure 17, 819-826, doi:10.1016/j.cardfail.2011.05.011 (2011). 
44 Conover, C. A. et al. Longevity and age-related pathology of mice deficient in pregnancyassociated plasma protein-A. The journals of gerontology. Series A, Biological sciences and medical sciences 65, 590-599, doi:10.1093/gerona/glq032 (2010).

45 Sebastian-Serrano, A. et al. Tissue-nonspecific Alkaline Phosphatase Regulates Purinergic Transmission in the Central Nervous System During Development and Disease. Computational and structural biotechnology journal 13, 95-100, doi:10.1016/j.csbj.2014.12.004 (2015).

46 Diaz-Hernandez, M. et al. Tissue-nonspecific alkaline phosphatase promotes the neurotoxicity effect of extracellular tau. The Journal of biological chemistry 285, 3253932548, doi:10.1074/jbc.M110.145003 (2010).

47 Vardy, E. R., Kellett, K. A., Cocklin, S. L. \& Hooper, N. M. Alkaline phosphatase is increased in both brain and plasma in Alzheimer's disease. Neuro-degenerative diseases $\mathbf{9}, 31-37$, doi:10.1159/000329722 (2012).

48 Kellett, K. A., Williams, J., Vardy, E. R., Smith, A. D. \& Hooper, N. M. Plasma alkaline phosphatase is elevated in Alzheimer's disease and inversely correlates with cognitive function. International journal of molecular epidemiology and genetics 2, 114-121 (2011).

49 Searles Quick, V. B., Davis, J. M., Olincy, A. \& Sikela, J. M. DUF1220 copy number is associated with schizophrenia risk and severity: implications for understanding autism and schizophrenia as related diseases. Translational psychiatry 5, e697, doi:10.1038/tp.2015.192 (2015).

50 Figueiro-Silva, J. et al. Neuronal pentraxin 1 negatively regulates excitatory synapse density and synaptic plasticity. The Journal of neuroscience : the official journal of the Society for Neuroscience 35, 5504-5521, doi:10.1523/JNEUROSCI.2548-14.2015 (2015). 
Satizabal et al.

51 Abad, M. A., Enguita, M., DeGregorio-Rocasolano, N., Ferrer, I. \& Trullas, R. Neuronal pentraxin 1 contributes to the neuronal damage evoked by amyloid-beta and is overexpressed in dystrophic neurites in Alzheimer's brain. The Journal of neuroscience : the official journal of the Society for Neuroscience 26, 12735-12747, doi:10.1523/JNEUROSCI.0575-06.2006 (2006).

52 Kulahin, N. et al. Structural model and trans-interaction of the entire ectodomain of the olfactory cell adhesion molecule. Structure 19, 203-211, doi:10.1016/j.str.2010.12.014 (2011).

53 Leshchyns'ka, I. et al. Abeta-dependent reduction of NCAM2-mediated synaptic adhesion contributes to synapse loss in Alzheimer's disease. Nature communications 6, 8836, doi:10.1038/ncomms9836 (2015).

54 Tobaben, S., Varoqueaux, F., Brose, N., Stahl, B. \& Meyer, G. A brain-specific isoform of small glutamine-rich tetratricopeptide repeat-containing protein binds to $\mathrm{Hsc70}$ and the cysteine string protein. The Journal of biological chemistry 278, 38376-38383, doi:10.1074/jbc.M301558200 (2003).

55 Fonte, V. et al. Interaction of intracellular beta amyloid peptide with chaperone proteins. Proceedings of the National Academy of Sciences of the United States of America 99, 94399444, doi:10.1073/pnas.152313999 (2002).

56 Zhu, J., Shang, Y. \& Zhang, M. Mechanistic basis of MAGUK-organized complexes in synaptic development and signalling. Nature reviews. Neuroscience 17, 209-223, doi:10.1038/nrn.2016.18 (2016).

57 Ingason, A. et al. Expression analysis in a rat psychosis model identifies novel candidate genes validated in a large case-control sample of schizophrenia. Translational psychiatry $\mathbf{5}$, e656, doi:10.1038/tp.2015.151 (2015). 
Satizabal et al.

58 Nithianantharajah, J. et al. Synaptic scaffold evolution generated components of vertebrate cognitive complexity. Nature neuroscience 16, 16-24, doi:10.1038/nn.3276 (2013).

59 Nalls, M. A. et al. Large-scale meta-analysis of genome-wide association data identifies six new risk loci for Parkinson's disease. Nature genetics 46, 989-993, doi:10.1038/ng.3043 (2014).

60 Guan, J. J. et al. DRAM1 regulates apoptosis through increasing protein levels and lysosomal localization of BAX. Cell death \& disease 6, e1624, doi:10.1038/cddis.2014.546 (2015).

61 Yu, M., Jiang, Y., Feng, Q., Ouyang, Y. \& Gan, J. DRAM1 protects neuroblastoma cells from oxygen-glucose deprivation/reperfusion-induced injury via autophagy. International journal of molecular sciences 15, 19253-19264, doi:10.3390/ijms151019253 (2014).

62 Scarpa, J. R. et al. Systems Genetic Analyses Highlight a TGFbeta-FOX03 Dependent Striatal Astrocyte Network Conserved across Species and Associated with Stress, Sleep, and Huntington's Disease. PLoS genetics 12, e1006137, doi:10.1371/journal.pgen.1006137 (2016).

63 Donlon, T. A. et al. F0X03 longevity interactome on chromosome 6. Aging cell, doi:10.1111/acel.12625 (2017).

64 Sears, J. C. \& Broihier, H. T. FoxO regulates microtubule dynamics and polarity to promote dendrite branching in Drosophila sensory neurons. Developmental biology 418, 40-54, doi:10.1016/j.ydbio.2016.08.018 (2016).

65 Peng, K. et al. Knockdown of Fox03a induces increased neuronal apoptosis during embryonic development in zebrafish. Neuroscience letters 484, 98-103, doi:10.1016/j.neulet.2010.07.068 (2010).

66 Elliott, L. et al. The genetic basis of human brain structure and function: 1,262 genome-wide associations found from 3,144 GWAS of multimodal brain imaging phenotypes from 9,707 
Satizabal et al.

UK Biobank participants. bioRxiv (2017).

<http://www.biorxiv.org/content/biorxiv/early/2017/08/21/178806.full.pdf>.

67 Santama, N., Er, C. P., Ong, L. L. \& Yu, H. Distribution and functions of kinectin isoforms. Journal of cell science 117, 4537-4549, doi:10.1242/jcs.01326 (2004).

68 Liu, X. A., Rizzo, V. \& Puthanveettil, S. V. Pathologies of Axonal Transport in Neurodegenerative Diseases. Translational neuroscience 3, 355-372, doi:10.2478/s13380012-0044-7 (2012).

69 Consortium, E. et al. Genome-wide association analysis of genetic generalized epilepsies implicates susceptibility loci at 1q43, 2p16.1, 2q22.3 and 17q21.32. Human molecular genetics 21, 5359-5372, doi:10.1093/hmg/dds373 (2012).

70 Martins-de-Souza, D. et al. Proteomic analysis identifies dysfunction in cellular transport, energy, and protein metabolism in different brain regions of atypical frontotemporal lobar degeneration. Journal of proteome research 11, 2533-2543, doi:10.1021/pr2012279 (2012).

71 Shulman, J. M. et al. Functional screening in Drosophila identifies Alzheimer's disease susceptibility genes and implicates Tau-mediated mechanisms. Human molecular genetics 23, 870-877, doi:10.1093/hmg/ddt478 (2014).

72 Yang, J. et al. Voxelwise meta-analysis of gray matter anomalies in Alzheimer's disease and mild cognitive impairment using anatomic likelihood estimation. Journal of the neurological sciences 316, 21-29, doi:10.1016/j.jns.2012.02.010 (2012).

73 den Heijer, T. et al. Hippocampal, amygdalar, and global brain atrophy in different apolipoprotein E genotypes. Neurology 59, 746-748 (2002).

74 de Jong, L. W. et al. Strongly reduced volumes of putamen and thalamus in Alzheimer's disease: an MRI study. Brain : a journal of neurology 131, 3277-3285, doi:10.1093/brain/awn 278 (2008). 
Satizabal et al.

75 Abramovic, L. et al. The association of antipsychotic medication and lithium with brain measures in patients with bipolar disorder. European neuropsychopharmacology: the journal of the European College of Neuropsychopharmacology 26, 1741-1751, doi:10.1016/j.euroneuro.2016.09.371 (2016).

76 Haller, S. et al. Combined analysis of grey matter voxel-based morphometry and white matter tract-based spatial statistics in late-life bipolar disorder. Journal of psychiatry \& neuroscience : JPN 36, 391-401, doi:10.1503/jpn.100140 (2011). 
Table 1. Genome-wide and probable* association results for subcortical brain volumes in the discovery meta-analysis in more than 25,000 Europeans from CHARGE and ENIGMA, and replication results in more than 9,000 Europeans from the UKBB

\begin{tabular}{|c|c|c|c|c|c|c|c|c|c|c|c|c|c|}
\hline \multirow{2}{*}{ SNP } & \multirow{2}{*}{ Chr } & \multirow{2}{*}{ Position } & \multirow{2}{*}{ Function } & \multirow{2}{*}{ Gene annotation } & \multirow{2}{*}{ A1/A2 } & \multicolumn{4}{|c|}{ Discovery in CHARGE and ENIGMA $\dagger$} & \multicolumn{4}{|c|}{ Replication in the UKBB $¥$} \\
\hline & & & & & & Freq. (A1) & Weight & Z-score & $\mathbf{P}$ & Freq. (A1) & Weight & Z-score & $\mathbf{P}$ \\
\hline \multicolumn{14}{|c|}{ Nucleus accumbens } \\
\hline rs11747514 & 5 & 65839259 & intergenic & MAST4 (dist=52kb) & $\mathrm{T} / \mathrm{G}$ & 0.22 & 23,683 & -5.44 & $5.34 \mathrm{E}-08$ & 0.23 & 9,409 & -1.28 & 0.201 \\
\hline rs145293717 & 3 & 190642692 & intergenic & SNAR-I (dist $=46 \mathrm{~kb})$ & $\mathrm{T} / \mathrm{G}$ & 0.09 & 23,360 & -5.32 & $1.04 \mathrm{E}-07$ & 0.09 & 9,409 & -3.33 & $8.61 \mathrm{E}-04$ \\
\hline \multicolumn{14}{|l|}{ Amygdala } \\
\hline rs953755 & 2 & 60255546 & intergenic & MIR4432 (dist $=358 \mathrm{~kb})$ & $\mathrm{T} / \mathrm{C}$ & 0.62 & 25,400 & -5.553 & $2.81 \mathrm{E}-08$ & 0.63 & 9,403 & -0.30 & 0.761 \\
\hline rs11111293 & 12 & 102921296 & intergenic & $I G F 1($ dist $=46 \mathrm{~kb})$ & $\mathrm{T} / \mathrm{C}$ & 0.78 & 25,434 & 5.195 & $2.05 \mathrm{E}-07$ & 0.78 & 9,403 & 0.72 & 0.469 \\
\hline rs429358 & 19 & 45411941 & missense & $A P O E$ & $\mathrm{~T} / \mathrm{C}$ & 0.85 & 24,549 & 5.127 & $2.94 \mathrm{E}-07$ & 0.85 & 9,403 & 0.41 & 0.681 \\
\hline \multicolumn{14}{|l|}{ Brainstem } \\
\hline rs11111090 & 12 & 102326461 & intergenic & $D R A M 1($ dist $=9 \mathrm{~kb})$ & $\mathrm{A} / \mathrm{C}$ & 0.52 & 19,930 & 8.706 & $3.14 \mathrm{E}-18$ & 0.51 & 9,400 & 5.46 & $4.72 \mathrm{E}-08$ \\
\hline rs1405 & 9 & 118954624 & intronic & PAPPA & $\mathrm{A} / \mathrm{G}$ & 0.39 & 19,930 & 8.482 & $2.22 \mathrm{E}-17$ & 0.39 & 9,400 & 5.89 & 3.93E-09 \\
\hline rs1549192 & 5 & 64965900 & 3'-UTR & $S G T B$ & $\mathrm{~T} / \mathrm{C}$ & 0.74 & 19,930 & -7.092 & $1.32 \mathrm{E}-12$ & 0.74 & 9,400 & -3.38 & $7.24 \mathrm{E}-04$ \\
\hline rs10792032 & 11 & 68984602 & intergenic & $M Y E O V($ dist $=77 \mathrm{~kb})$ & $\mathrm{A} / \mathrm{G}$ & 0.48 & 19,769 & 6.127 & $8.98 \mathrm{E}-10$ & 0.49 & 9,400 & -4.45 & $8.53 \mathrm{E}-06$ \\
\hline rs201287891 & 16 & 52867262 & intergenic & CHD9 $($ dist $=221 \mathrm{~kb})$ & $\mathrm{D} / \mathrm{I}$ & 0.37 & 19,205 & 6.082 & $1.18 \mathrm{E}-09$ & NA & NA & NA & NA \\
\hline rs9398173 & 6 & 109000316 & intronic & FOXO3 & $\mathrm{T} / \mathrm{C}$ & 0.34 & 19,930 & -6.058 & $1.38 \mathrm{E}-09$ & 0.29 & 9,400 & -2.81 & 4.95E-03 \\
\hline rs112994922 & 6 & 149919887 & intronic & KATNA1 & $\mathrm{D} / \mathrm{I}$ & 0.32 & 18,552 & 5.65 & $1.60 \mathrm{E}-08$ & NA & NA & NA & NA \\
\hline rs201708769 & 20 & 49127281 & intronic & PTPN1 & $\mathrm{D} / \mathrm{I}$ & 0.21 & 19,205 & -5.597 & $2.18 \mathrm{E}-08$ & NA & NA & NA & NA \\
\hline rs11934535 & 4 & 94936015 & intergenic & ATOH1 $($ dist $=184 \mathrm{~kb})$ & $\mathrm{A} / \mathrm{G}$ & 0.60 & 19,930 & -5.59 & $2.28 \mathrm{E}-08$ & 0.58 & 9,400 & -0.99 & 0.322 \\
\hline rs12479469 & 20 & 61145196 & nc transcript & C20orf166-AS1 & $\mathrm{A} / \mathrm{G}$ & 0.33 & 16,943 & -5.489 & 4.05E-08 & 0.34 & 9,400 & -2.68 & 7.27E-03 \\
\hline \multicolumn{14}{|c|}{ Caudate nucleus } \\
\hline rs2845878 & 11 & 92019253 & intergenic & FAT3 (dist $=28 \mathrm{~kb})$ & $C / G$ & 0.33 & 25,563 & -6.464 & $1.02 \mathrm{E}-10$ & 0.33 & 9,400 & -6.50 & $7.80 \mathrm{E}-11$ \\
\hline rs888234 & 9 & 128880042 & intergenic & $P B X 3($ dist $=150 \mathrm{~kb})$ & $\mathrm{A} / \mathrm{G}$ & 0.58 & 25,449 & -6.001 & $1.96 \mathrm{E}-09$ & 0.59 & 9,400 & -3.05 & $2.27 \mathrm{E}-03$ \\
\hline rs7584428 & 2 & 45128493 & intergenic & SIX3 (dist $=40 \mathrm{~kb})$ & $\mathrm{A} / \mathrm{G}$ & 0.40 & 25,563 & -5.623 & $1.88 \mathrm{E}-08$ & 0.42 & 9,400 & -1.67 & 0.096 \\
\hline rs76099988 & 9 & 98329371 & intergenic & PTCH1 (dist=97kb) & $\mathrm{A} / \mathrm{T}$ & 0.08 & 25,445 & 5.599 & $2.15 \mathrm{E}-08$ & 0.09 & 9,400 & 1.20 & 0.231 \\
\hline \multicolumn{14}{|c|}{ Globus pallidus } \\
\hline rs148470213 & 14 & 56193700 & intergenic & KTN1 (dist $=42 \mathrm{~kb}$ ) & $\mathrm{T} / \mathrm{C}$ & 0.54 & 25,534 & 7.058 & $1.69 \mathrm{E}-12$ & 0.56 & 9,352 & 1.97 & $4.92 \mathrm{E}-02$ \\
\hline rs1349470 & 8 & 42430502 & intergenic & SMIM19 $($ dist $=22 \mathrm{~kb})$ & $\mathrm{A} / \mathrm{G}$ & 0.58 & 25,534 & 6.536 & $6.31 \mathrm{E}-11$ & 0.59 & 9,352 & 9.03 & $1.65 \mathrm{E}-19$ \\
\hline rs12128419 & 1 & 21864879 & intronic & $A L P L$ & $\mathrm{~T} / \mathrm{C}$ & 0.67 & 25,335 & -5.561 & $2.68 \mathrm{E}-08$ & 0.69 & 9,352 & -3.37 & 7.41E-04 \\
\hline rs182599518 & 14 & 103980792 & intergenic & $C K B($ dist $=52 \mathrm{~kb})$ & $\mathrm{T} / \mathrm{C}$ & 0.99 & 2,142 & -5.456 & $4.87 \mathrm{E}-08$ & 1.00 & 9,352 & -0.49 & 0.627 \\
\hline
\end{tabular}

Putamen 


\begin{tabular}{|c|c|c|c|c|c|c|c|c|c|c|c|c|c|}
\hline rs8017172 & 14 & 56199048 & intergenic & KTN1 (dist $=47 \mathrm{~kb})$ & $\mathrm{A} / \mathrm{G}$ & 0.42 & 25,393 & -12.137 & $6.69 \mathrm{E}-34$ & 0.42 & 9,402 & -7.60 & $3.01 \mathrm{E}-14$ \\
\hline rs62097986 & 18 & 50818827 & intronic & $D C C$ & $\mathrm{~A} / \mathrm{C}$ & 0.44 & 25,393 & 7.406 & $1.31 \mathrm{E}-13$ & 0.42 & 9,402 & 6.22 & $5.13 \mathrm{E}-10$ \\
\hline rs1484994 & 20 & 30305975 & intronic & $B C L 2 L 1$ & $\mathrm{~A} / \mathrm{G}$ & 0.71 & 24,113 & 7.072 & $1.52 \mathrm{E}-12$ & 0.71 & 9,402 & 4.62 & $3.79 \mathrm{E}-06$ \\
\hline rs512556 & 11 & 83288085 & intronic & $D L G 2$ & $\mathrm{~A} / \mathrm{C}$ & 0.64 & 25,393 & -6.857 & $7.06 \mathrm{E}-12$ & 0.62 & 9,402 & -3.84 & $1.23 \mathrm{E}-04$ \\
\hline rs597583 & 11 & 117421799 & intronic & DSCAML1 & $\mathrm{C} / \mathrm{G}$ & 0.80 & 25,393 & 6.54 & $6.14 \mathrm{E}-11$ & 0.80 & 9,402 & 2.16 & $3.10 \mathrm{E}-02$ \\
\hline \multicolumn{14}{|l|}{ Thalamus } \\
\hline rs144443274 & 17 & 78449948 & missense & NPTX1 & $\mathrm{T} / \mathrm{C}$ & 0.18 & 22,864 & -6.172 & $6.73 \mathrm{E}-10$ & 0.20 & 9,412 & -2.37 & $1.77 \mathrm{E}-02$ \\
\hline rs66562752 & 21 & 22530867 & intronic & NCAM2 & $\mathrm{A} / \mathrm{C}$ & 0.57 & 25,585 & 5.623 & $1.88 \mathrm{E}-08$ & 0.58 & 9,412 & -2.29 & $2.21 \mathrm{E}-02$ \\
\hline rs8045946 & 16 & 68779469 & intronic & $C D H 1$ & $\mathrm{~A} / \mathrm{G}$ & 0.80 & 2,447 & -5.518 & $3.43 \mathrm{E}-08$ & NA & NA & NA & NA \\
\hline rs143943992 & 14 & 66534309 & intergenic & FUT8 (dist=418kb) & $\mathrm{A} / \mathrm{G}$ & 0.01 & 1,058 & 5.497 & $3.85 \mathrm{E}-08$ & 0.01 & 9,412 & -0.79 & 0.429 \\
\hline
\end{tabular}

Chr = chromosome; Freq. = frequency of the coded allele; dist = distance from nearest gene; A1 = coded allele; A2 = non-coded allele

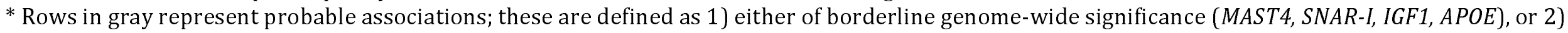
infrequent variants reliably genotyped in $\mathrm{n}<2,500$ individuals $(C K B, C D H 1, F U T 8)$.

$\uparrow$ GWA analyses are adjusted for sex, age, age ${ }^{2}$, total intracranial volume and population stratification

¥ GWA analyses are adjusted for sex, age, age ${ }^{2}$, total brain volume and population stratification. UKBB results for proxy SNPs as follows:

$\mathrm{rs} 148470213 \sim \mathrm{rs} 1959089\left(\mathrm{r}^{2}=.48, \mathrm{C}=\mathrm{C}, \mathrm{T}=\mathrm{T}\right) ; \mathrm{rs} 182599518 \sim \mathrm{rs} 145525075\left(\mathrm{r}^{2}=1, \mathrm{~T}=\mathrm{C}, \mathrm{C}=\mathrm{T}\right) ; \mathrm{rs} 144443274 \sim \mathrm{rs} 34481566\left(\mathrm{r}^{2}=.78, \mathrm{C}=\mathrm{C}, \mathrm{T}=\mathrm{T}\right)$;

$\operatorname{rs} 145293717 \sim \operatorname{rs} 34481566\left(r^{2}=1, G=G, T=A\right) ; r s 138074335 \sim r s 8756\left(r^{2}=1, A=C, G=A\right)$ 
Figure 1. Heritability and Manhattan plot of genetic variation associated with subcortical brain volumes in the discovery sample. Analyses were adjusted for sex, age, age ${ }^{2}$, total intracranial volume, and population structure. A. Heritability (h ${ }^{2}$ ) estimates were performed with SOLAR in the Framingham Heart Study ( $n=895)$ and the Austrian Stroke Prevention-Family Study ( $=370)$. B. Combined Manhattan plot. Each dot denotes a single genetic variant plotted according to its genomic position ( $\mathrm{x}$-axis) and $-\log 10(\mathrm{P})$ for the associations with each subcortical volume ( $\mathrm{y}$-axis). Variants are colored differently for each structure (see legend in A). The solid horizontal line denotes genome-wide significance $\left(\mathrm{P}<5 \times 10^{-8}\right)$, the dashed horizontal line denotes a threshold of $\mathrm{P}<10^{-6}$. Individual Manhattan plots may be found in the Supplementary note. 
Satizabal et al.

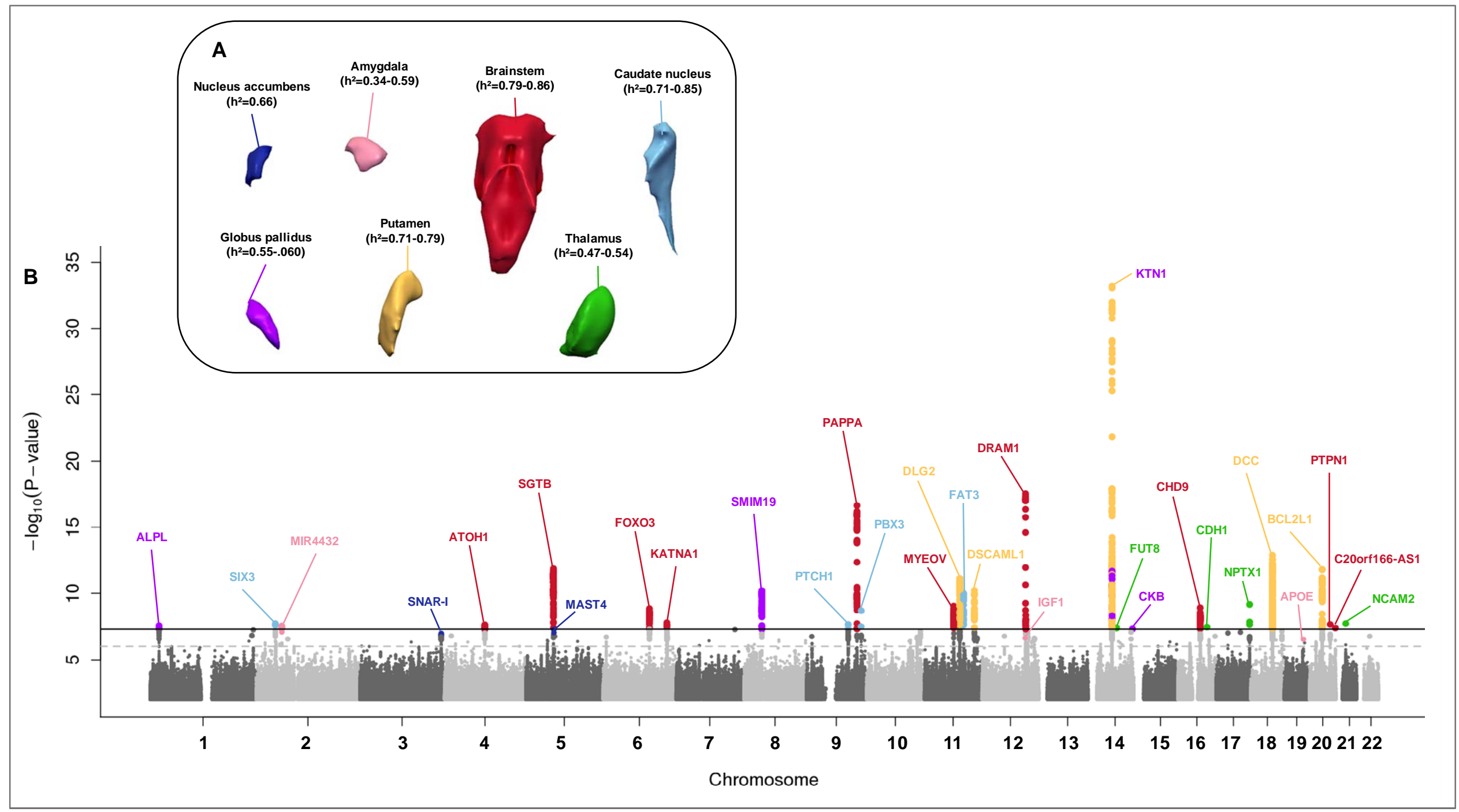

48 
Satizabal et al.

Figure 2. Genetic and phenotypic correlations. In this heat map, the size of the circle is proportional to the strength of correlation $(\rho)$ and the direction is presented in the color label on the bottom; ' $X$ ' indicates no significant association ( $p>0.05$ ). (A) Partial phenotypic (upper triangle) and genetic (lower) correlations among the subcortical structures included in this report. Partial phenotypic correlations were derived from the subcortical volumes of $n=894$ participants from the Framingham Heart Study, adjusting for sex, age, age $^{2}$, total intracranial volume and PC1. (B) Genetic correlations using LD score regression between subcortical brain volumes and other MRI-derived volumes, anthropometric, and neuropsychiatric traits.

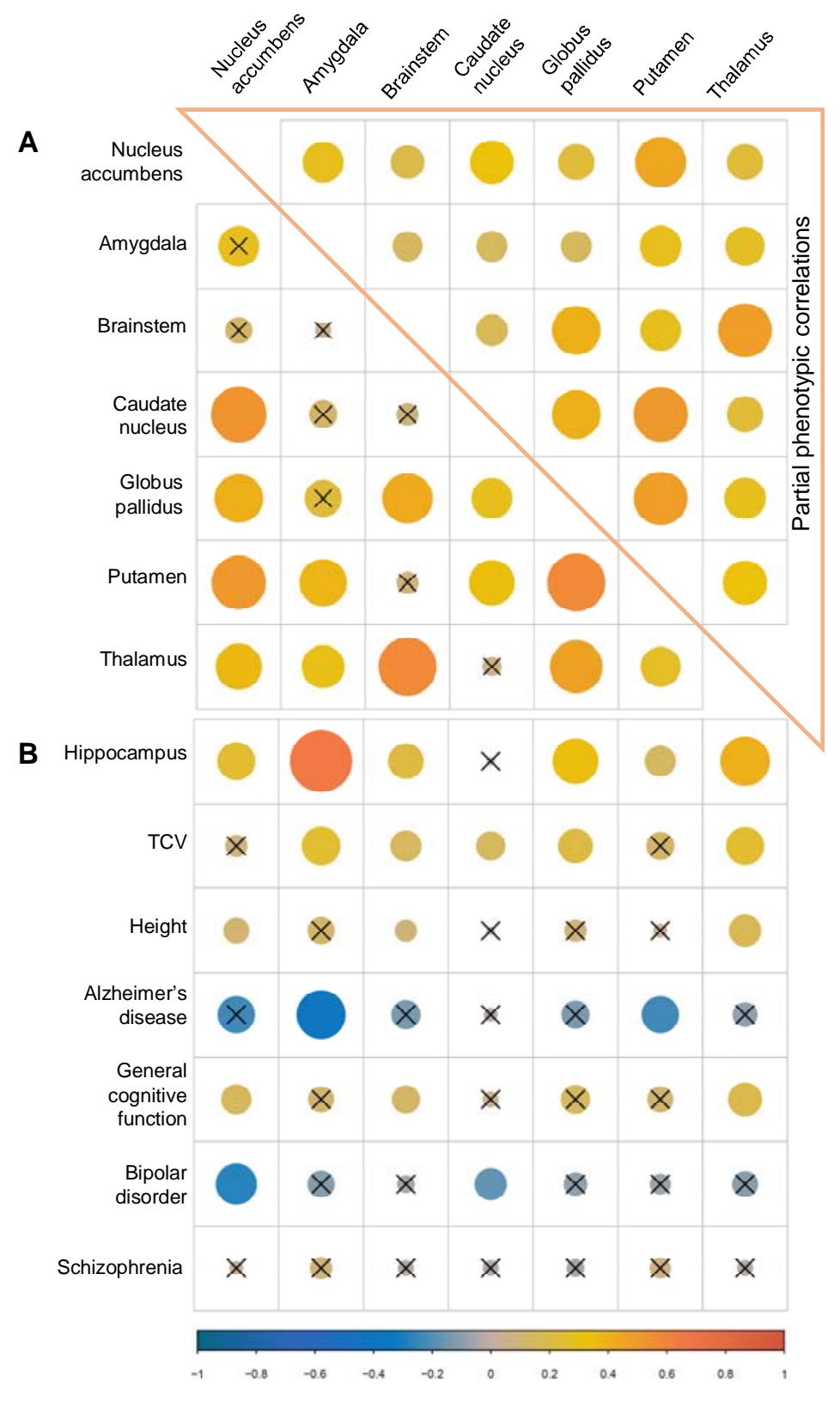




\section{Figure 3. Protein-protein interaction network of 57 genes enriched for common variants}

\section{influencing the volume of subcortical structures using medium-confidence interaction} scores from the human STRING database. The edges represent protein-protein associations, where the edge color indicates the predicted mode of action and the edge shape the predicted action effects (see labels on the bottom). Colored nodes represent the queried proteins and first shell of interactors (5 maximum), whereas white nodes represent the second shell of interactors (5 maximum).

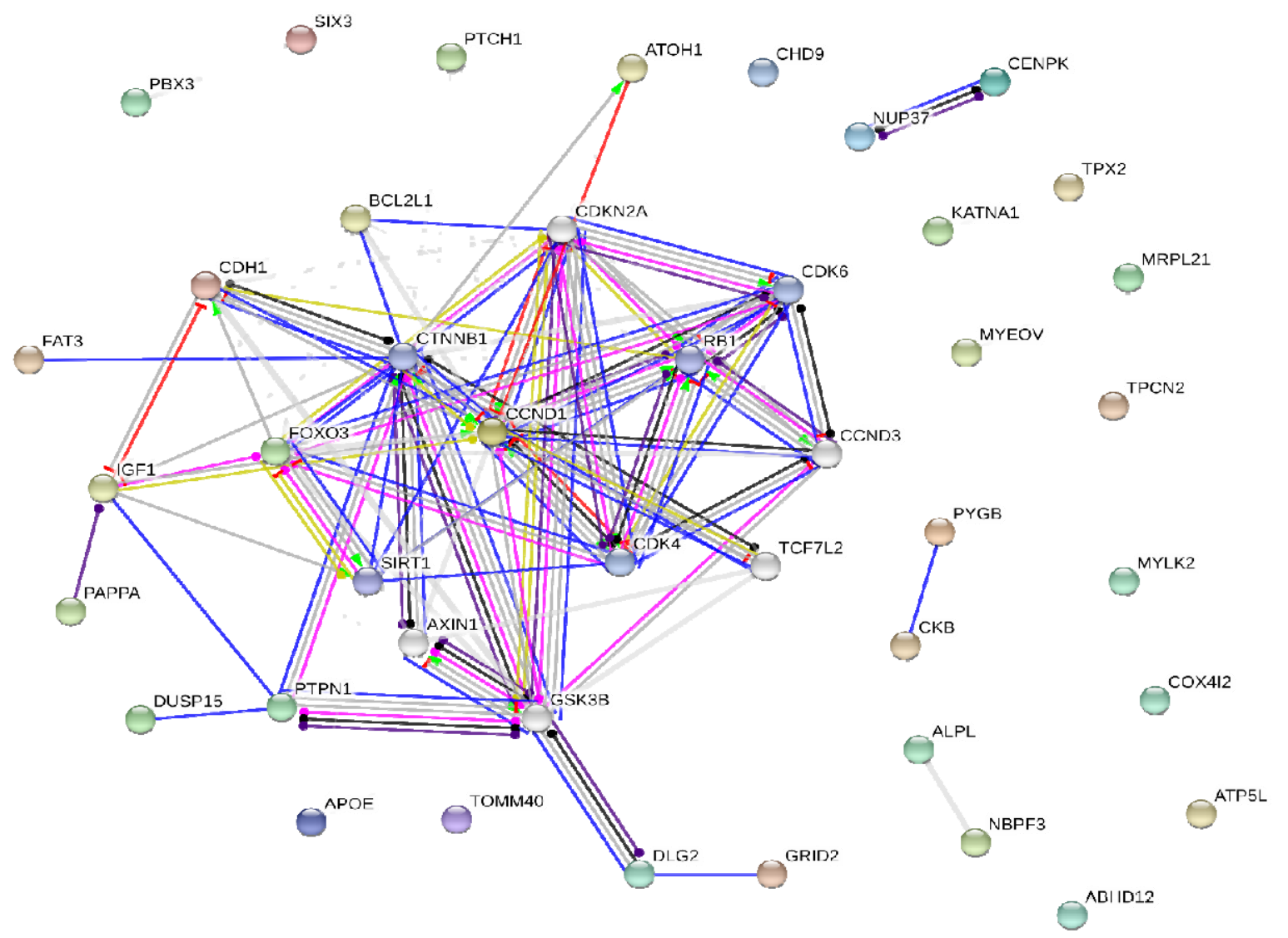

Predicted mode of action

O-Oactivation

- posttranslational modification

-O inhibition

$\mathrm{O}-\mathrm{binding}$

- - catalysis

- - phenotype

$\mathrm{O}-\mathrm{reaction}$

- - transcriptional regulation
Predicted action effects

$O \rightarrow O$ positive

$0 \rightarrow O$ negative

$\mathrm{O}-\mathrm{O}$ unspecified 